\title{
TOPOLOGICAL PROPERTIES OF SPACES ADMITTING FREE GROUP ACTIONS
}

\author{
ROSS GEOGHEGAN AND CRAIG R. GUILBAULT
}

\begin{abstract}
In 1992, David Wright proved a remarkable theorem about which contractible open manifolds are covering spaces. He showed that if a one-ended open manifold $M^{n}$ has pro-monomorphic fundamental group at infinity which is not protrivial and is not stably $\mathbb{Z}$, then $M$ does not cover any manifold (except itself). In the non-manifold case, Wright's method showed that when a one-ended, simply connected, locally compact ANR $X$ with pro-monomorphic fundamental group at infinity admits an action of $\mathbb{Z}$ by covering transformations then the fundamental group at infinity of $X$ is (up to pro-isomorphism) an inverse sequence of finitely generated free groups. We improve upon this latter result, by showing that $X$ must have a stable finitely generated free fundamental group at infinity. Simple examples show that a free group of any finite rank is possible.

We also prove that if $X$ (as above), admits a non-cocompact action of $\mathbb{Z} \times \mathbb{Z}$ by covering transformations, then $X$ is simply connected at infinity.

We deduce the following corollary in group theory: Every finitely presented oneended group $G$ which contains an element of infinite order satisfies exactly one of the following:

- $G$ is simply connected at infinity;

- $G$ is virtually a surface group;

- The fundamental group at infinity of $G$ is not pro-monomorphic.

Our methods also provide a quick new proof of Wright's open manifold theorem.
\end{abstract}

\section{INTRODUCTION}

In this paper we address a series of questions which are of interest in both topology and geometric group theory.

Let $X$ be a locally finite simply connected one-ended CW complex (or, more generally, a locally compact simply connected one-ended metric ANR). We are interested in the fundamental group at infinity ${ }^{1}$ of $X$, and what special properties it must have in order that $X$ can be a non-trivial covering space. In particular, we consider:

Question 1: Does $\mathbb{Z}$ act as a group of covering transformations on $X$ ?

Date: October 15, 2011.

1991 Mathematics Subject Classification. Primary 57M10; Secondary 57S30, 57M07, 20F65.

Key words and phrases. pro-monomorphic, stably free, fundamental group at infinity, Wright's Theorem.

This project was aided by a Simons Foundation Collaboration Grant awarded to the second author.

${ }^{1}$ This and several other concepts mentioned in the introduction will be discussed in detail later in the paper. 
Question 2: Does some group $G$ act cocompactly as a group of covering transformations on $X$ ?

Turning the latter question around, let $K$ be a compact ANR (for example, a finite complex) with one-ended universal cover $X$. Can the fundamental group at infinity of $X$ be arbitrary? Or how restricted is it?

Here are closely related manifold versions of our questions:

Question 3: If $M^{n}$ is a contractible open manifold which is not homeomorphic to $\mathbb{R}^{n}$ does it cover any manifold non-trivially? In other words, does $M^{n}$ support a properly discontinuous free action of a non-trivial (necessarily torsion free) group?

Question 4: Must the universal cover of a closed aspherical n-manifold be homeomorphic to $\mathbb{R}^{n}$ ?

We are certainly not the first authors to address these questions. We will begin by reviewing some of what is known, starting with the manifold case.

\section{Concerning Question 4}

In dimensions $\leq 3$ the universal cover of a closed aspherical manifold is indeed homeomorphic to a Euclidean space. This is classical in dimensions $\leq 2$ and follows from Perelman's solution to the Poincaré Conjecture in dimension 3. A negative answer was obtained by Davis $[\mathrm{Da}$ in all dimensions $\geq 4$. The invariant which detects Davis's examples is the fundamental group at infinity; specifically, Davis gave examples of a one-ended Coxeter group $\Gamma$ having a subgroup $G$ of finite index such that the universal cover of a closed manifold $K(G, 1)$ is not simply connected at infinity.

\section{Concerning Question 3}

Recall that when $n \geq 3$ a contractible open $n$-manifold is homeomorphic to $\mathbb{R}^{n}$ if and only if it is simply connected at infinity. (This will be discussed further in \$6.) So one looks for an answer among contractible manifolds which are not simply connected at infinity (i.e. with non-trivial fundamental group at infinity). Whitehead [Wh] gave the first example of a contractible open 3-manifold which is not simply connected at infinity. Later, it was shown in $[\mathrm{Mc}$ that uncountably many pairwise non-homeomorphic such 3-manifolds exist. But until the 1980's it was not known whether any of these could cover a manifold non-trivially.

Myers [My1 obtained the first notable result along these lines, proving that no member of a certain class of Whitehead-type contractible open 3-manifolds admits an action of $\mathbb{Z}$ by covering transformations. Later, Wright $[\mathrm{Wr}$ gave a significant generalization by showing that a contractible open $n$-manifold $(n \geq 3)$ with pro-monomorphi2 2 fundamental group at infinity, which admits a nontrivial action by covering transformations, is necessarily simply connected at infinity. All the aforementioned Whitehead-type 3-manifolds have this pro-monomorphic property, hence they do not

\footnotetext{
${ }^{2}$ This is defined in $2_{2}$ roughly, it means that the inverse sequence of fundamental groups of complements of larger and larger compact submanifolds looks like a sequence of monomorphisms.
} 
cover manifolds non-trivially. Wright's theorem also implies that the interiors of compact contractible $n$-manifolds with non-simply connected boundaries do not cover non-trivially.

\section{Concerning Question 1}

Wright's method extends beyond manifold topology3. In particular, he proved:

Theorem 1.1 ([Wr, Th.9.1]). Let $X$ be a simply connected one-ended locally compact $A N R$, and assume $X$ has pro-monomorphic fundamental group at infinity. If $\mathbb{Z}$ acts as covering transformations on $X$ then the fundamental group at infinity of $X$ is pro-free and pro-finitely generated.

For this version of Wright's Theorem, see [Ge, Th.16.3.4].

The conclusion of Theorem 1.1 implies that the fundamental group at infinity may be represented by

$$
F_{1} \leftarrow F_{2} \longleftarrow F_{3} \leftarrow \cdots
$$

where each $F_{i}$ is a finitely generated free group. Given the pro-monomorphic hypothesis, one may also assume that the bonding homomorphisms in this sequence are injective. The main theorem of this paper is an improvement of Wright's Theorem 1.1. namely:

Theorem 1.2. Let $X$ be a simply connected one-ended locally compact ANR with promonomorphic fundamental group at infinity. If $\mathbb{Z}$ acts as covering transformations on $X$, then the fundamental group at infinity of $X$ is stably a finitely generated free group.

The conclusion of Theorem 1.2 means that the finitely generated free groups $F_{i}$ all can be taken to have the same rank, and the bonding morphisms can be taken to be isomorphisms.

Remark 1. "Stable" means, roughly, that the inverse sequence of fundamental groups of complements of larger and larger compacta looks like a sequence of isomorphisms. Thus "stable" is equivalent to "pro-monomorphic and pro-epimorphic". "Pro-epimorphic" is also known as "semistable" or "Mittag-Leffler". Thus Theorem 1.2 improves on Wright's Theorem by establishing semistability.

Example 1. As a simple illustration, let $X$ be a simply connected polyhedron with "solenoidal" fundamental group at infinity; i.e. having the form

$$
\mathbb{Z} \stackrel{\times 2}{\longleftarrow} \mathbb{Z} \stackrel{\times 2}{\longleftarrow} \mathbb{Z} \stackrel{\times 2}{\longleftarrow} \cdots .
$$

(Such spaces are easy to construct.) Our theorem prohibits $X$ from admitting an action of $\mathbb{Z}$ by covering transformations.

\footnotetext{
${ }^{3}$ Background and more detail concerning Wright's Theorem can be found in Section 16.3 of [Ge].
} 


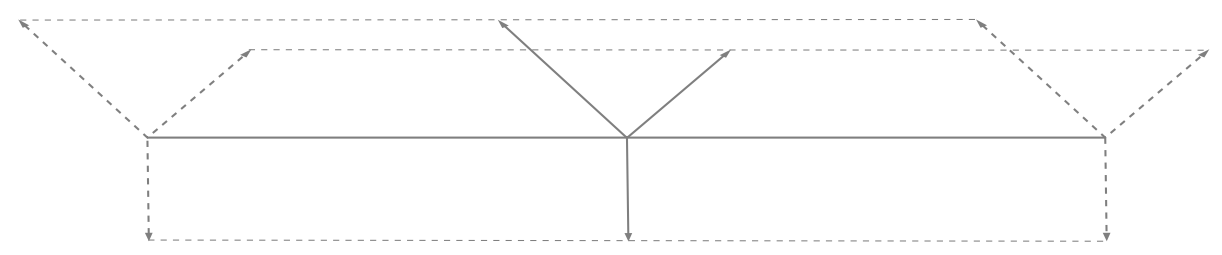

FiguRE $1 . K_{3} \times \mathbb{R}$.

On the other hand, there exist contractible spaces which have stable free fundamental groups at infinity of any finite rank, and which admit actions of $\mathbb{Z}$ by covering transformations:

Example 2. Let $K_{n}$ be a wedge of $n$ rays with a common vertex and let $X=K_{n} \times \mathbb{R}$. Then $X$ has a stable fundamental group at infinity that is free of rank $n-1$. Moreover, the homeomorphism $j: X \rightarrow X$ induced by a translation in the $\mathbb{R}$-factor generates an action of $\mathbb{Z}$ by covering transformations. As a variation, one may combine translation in the $\mathbb{R}$-coordinate with a permutation of the rays of $K_{n}$. By doing so, it is possible to obtain quotient spaces with different numbers of ends and various fundamental group behavior at those ends. These examples will be useful to keep in mind when reading \$3.

When $X$ admits a free $(\mathbb{Z} \times \mathbb{Z})$-action, we are able to prove a stronger result.

Theorem 1.3. Let $X$ be a simply connected one-ended locally compact ANR with pro-monomorphic fundamental group at infinity. If $X$ admits an action of $\mathbb{Z} \times \mathbb{Z}$ by covering transformations, then either that action is cocompact or $X$ is simply connected at infinity.

\section{Concerning Question 2}

Let $G$ be a finitely presented one-ended group having an element of infinite order, and let $K$ be a finite complex whose fundamental group is isomorphic to $G$. The universal cover $X$ of $K$ has fundamental group at infinity represented by an inverse sequence

$$
G_{1} \longleftarrow G_{2} \longleftarrow G_{3} \longleftarrow \cdots
$$

where each $G_{i}$ is the fundamental group of the complement of a compact subcomplex of $X$. No example is known where this sequence fails to be semistable. Easy examples occur where this sequence (up to pro-isomorphism) has or does not have promonomorphic fundamental group at infinity. Here, we only consider the case where $X$ has pro-monomorphic fundamental group at infinity. Theorem 1.2 implies that, letting $j$ be an element of infinite order, the infinite cyclic group $\langle j\rangle$ acts as covering transformations on $X$, hence $X$ has a stable finitely generated free fundamental group at infinity. In other words, one may assume the groups $G_{i}$ are all free and finitely generated of the same rank and the bonding morphisms are isomorphisms. But in 
this case more is known. As explained in the proof of [Ge, Th.16.5.6], for homological reasons a theorem of Farrell [Fa] implies that this rank must be either 0 or 1 . Moreover, in the rank 1 case, Bowditch [Bo] has shown that $G$ is virtually a surface group-meaning that $G$ contains a finite index subgroup which is the fundamental group of a closed surface. In other words, a consequence of our main theorem is:

Theorem 1.4. Let the one-ended finitely presented group $G$ have pro-monomorphic fundamental group at infinity and assume $G$ contains an element of infinite order. Then $G$ is either simply connected at infinity or $G$ is virtually a surface group.

Remark 2. The advance, here, over [Ge, Th.16.5.6] is that we do not have to make semistability of $G$ part of the hypothesis.

Remark 3. The one-ended examples of Davis, mentioned above, where $G$ is a torsion free subgroup of finite index in a suitable Coxeter group, show that the universal cover $X$ in Theorem 1.4 does not always have pro-monomorphic fundamental group at infinity when it is the universal cover of a finite complex.

A similar application of Theorem 1.2 applies to CAT(0) groups.

Theorem 1.5. If the $C A T(0)$ group $G$ acts geometrically on a one-ended proper $C A T(0)$ space $X$ such that $\partial_{\infty} X$ is 1-dimensional and $X$ has pro-monomorphic fundamental group at infinity, then $G$ is virtually a surface group.

Proof. [Sw, Th. 11] ensures the existence of an infinite order element in $G$, thus permitting an application of Theorem [1.2. Then [GO, Main Theorem] rules out the possibility of $X$ being simply connected at infinity when $\partial_{\infty} X$ is 1-dimensional.

This paper also includes some manifold theoretic results, most notably, a brief new proof of [Wr, Main Theorem] and a new proof of a theorem from [GM].

\section{A special case of the Borel Construction}

The starting point of our argument is the following useful observation:

Proposition 1.6. Let $Y$ be a connected, locally path connected space on which an infinite cyclic group $J=\langle j\rangle$ acts as covering transformations. Then $(J \backslash X) \times \mathbb{R}$ is homeomorphic to the mapping torus $T_{j}(X)$.

Remark 4. In [GM], Proposition 1.6 is proved using bundle theory; the authors cite an earlier paper by Farrell [Fa]. Another proof is given in $\mathrm{Ge}$ as Proposition 13.7.4. A new and elementary proof of Proposition 1.6 is contained within our proof of Theorem 1.2, see Remark 11 in $\oint 8$.

Proposition 1.6 provides two pictures of the same space. Those pictures yield canonical, but very different, families of neighborhoods of infinity-rectangular neighborhoods of infinity in the case of $(J \backslash X) \times \mathbb{R}$ and mapping torus neighborhoods of infinity in the case of $T_{j}(X)$ (see $₫ 3$ and $\S 4$ ). By comparing the fundamental group 
systems arising from these different pictures, we are sometimes able to coax out information about the original space $X$, as was done, for example, in [GM]. The delicate nature of that task accounts for much of the work found here.

\section{Layout}

The layout of this paper is as follows. In $\$ 2$ we provide most of the necessary definitions and background. The first portion of that section is purely algebraic - dealing primarily with inverse sequences of groups. In addition to essential definitions and notation, some basic results are proved for later use. The latter portion of 92 discusses the topology of noncompact spaces; neighborhoods of infinity and fundamental pro-groups are discussed and some useful equivalences are reviewed. In $₫ 3$ and $\$ 4$ we look at canonical neighborhoods of infinity in the homeomorphic spaces, $(J \backslash X) \times \mathbb{R}$ and $T_{j}(X)$, of Proposition 1.6. Of particular importance will be some clean descriptions of the fundamental groups of those neighborhoods of infinity. The remainder of the paper contain the proofs of our principal results. In $\$ 6$ we focus on manifolds. Our new proof of Wright's main theorem may be viewed as a warm-up for the more general (non-manifold) version that is Theorem 1.2, $\$ 7$ and $₫ 8$ are devoted to the proof of Theorem 1.2. In the final section we push our techniques one step further to prove Theorem 1.3 .

The authors wish to acknowledge the contribution of the referee, whose comments led to a significant simplification in the proof of our main theorem.

\section{Definitions And BaCKGround}

This section contains much of the terminology and notation needed for the remainder of the paper. In addition, several preliminary results are presented. The section is divided into two parts; the first is entirely algebraic - dealing primarily with inverse sequences of groups, while the second is topological-dealing primarily with the topology at the ends of noncompact spaces.

2.1. Algebra of inverse sequences. Throughout this subsection all arrows denote homomorphisms, while arrows of the type $\rightarrow$ or $\leftarrow$ denote surjections and arrows of the type $\longmapsto$ and $\longleftarrow$ denote injections. The symbol $\cong$ indicates an isomorphism.

Let

$$
G_{0} \stackrel{\lambda_{1}}{\longleftarrow} G_{1} \stackrel{\lambda_{2}}{\longleftarrow} G_{2} \stackrel{\lambda_{3}}{\longleftarrow} \ldots
$$

be an inverse sequence of groups. A subsequence of $\left\{G_{i}, \lambda_{i}\right\}$ is an inverse sequence of the form

$$
G_{i_{0}} \stackrel{\lambda_{i_{0}+1} \circ \cdots \circ \lambda_{i_{1}}}{\longleftarrow} G_{i_{1}} \stackrel{\lambda_{i_{1}+1} \circ \cdots \circ \lambda_{i_{2}}}{\longleftarrow} G_{i_{2}} \stackrel{\lambda_{i_{2}+1} \circ \cdots \circ \lambda_{i_{3}}}{\longleftarrow} \cdots .
$$

In the future we will denote a composition $\lambda_{i} \circ \cdots \circ \lambda_{j}(i \leq j)$ by $\lambda_{i, j}$. 
Sequences $\left\{G_{i}, \lambda_{i}\right\}$ and $\left\{H_{i}, \mu_{i}\right\}$ are pro-isomorphic if, after passing to subsequences, there exists a commuting "ladder diagram":

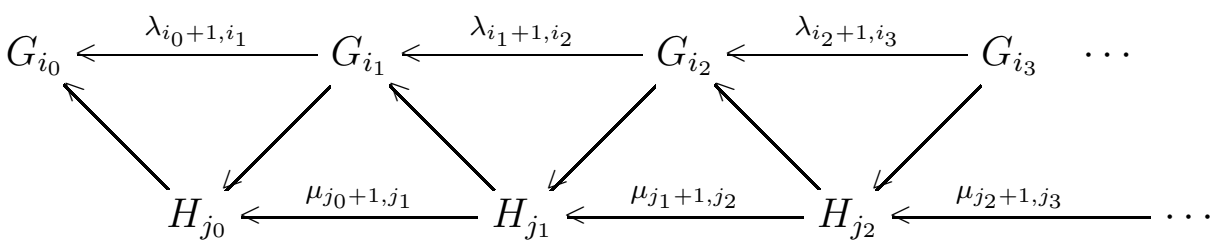

Clearly an inverse sequence is pro-isomorphic to any of its subsequences. To avoid tedious notation, we sometimes do not distinguish $\left\{G_{i}, \lambda_{i}\right\}$ from its subsequences. Instead we assume that $\left\{G_{i}, \lambda_{i}\right\}$ has the desired properties of a preferred subsequenceprefaced by the words "after passing to a subsequence and relabeling".

The inverse limit of a sequence $\left\{G_{i}, \lambda_{i}\right\}$ is a subgroup of $\prod G_{i}$ defined by

$$
\lim _{\longleftarrow}\left\{G_{i}, \lambda_{i}\right\}=\left\{\left(g_{0}, g_{1}, g_{2}, \cdots\right) \in \prod_{i=0}^{\infty} G_{i} \mid \lambda_{i}\left(g_{i}\right)=g_{i-1}\right\} .
$$

Notice that, for each $i$, there is a projection homomorphism $p_{i}: \lim _{\longleftarrow}\left\{G_{i}, \lambda_{i}\right\} \rightarrow G_{i}$. It is a standard fact that pro-isomorphic inverse sequences have isomorphic inverse limits.

An inverse sequence $\left\{G_{i}, \lambda_{i}\right\}$ is stable if it is pro-isomorphic to a constant inverse sequence $\left\{H, \operatorname{id}_{H}\right\}$, or equivalently, to an inverse sequence $\left\{H_{i}, \mu_{i}\right\}$ where each $\mu_{i}$ is an isomorphism. In those cases, the projection homomorphisms take $\lim _{\longleftarrow}\left\{G_{i}, \lambda_{i}\right\}$ isomorphically onto $H$ and each of the $H_{i}$.

Another condition equivalent to the stability of $\left\{G_{i}, \lambda_{i}\right\}$ is that, after passing to an appropriate subsequence and relabeling, there exists a commutative diagram of the form

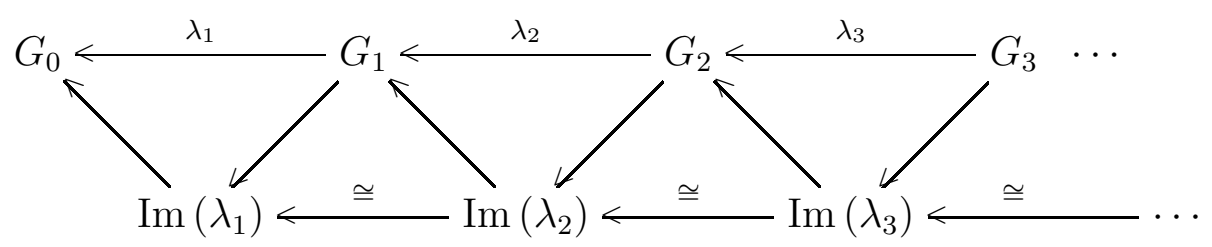

where all unlabeled homomorphisms are obtained by restriction or inclusion.

If $\left\{G_{i}, \lambda_{i}\right\}$ is pro-isomorphic to some $\left\{H_{i}, \mu_{i}\right\}$, where each $\mu_{i}$ is an epimorphism, we call $\left\{G_{i}, \lambda_{i}\right\}$ semistable (or Mittag-Leffler, or pro-epimorphic). In that case, there exists a ladder diagram of the type described in (2.2), but with the isomorphisms replaced by epimorphisms. Similarly, if $\left\{H_{i}, \mu_{i}\right\}$ can be chosen so that each $\mu_{i}$ is a monomorphism, we call our inverse sequence pro-monomorphic; in that case, there exists a diagram of type (2.2) for which maps in the bottom row are monomorphisms. It is easy to show that an inverse sequence that is both semistable and pro-monomorphic is stable.

Given a group $G$, a pair of isomorphic subgroups $H, H^{\prime} \leq G$, and a specified isomorphism $\varphi: H \rightarrow H^{\prime}$, the $H N N$ extension of $G$ by $\varphi$ is the group

$$
G *_{\varphi}=\left\langle G, t \mid t^{-1} h t=\varphi(h) \forall h \in H\right\rangle .
$$


In this setup, $G$ is called the base group, $H$ and $H^{\prime}$ the associated subgroups, and $t$ the stable letter. It is a standard fact that $G$ injects into $G *_{\varphi}$ (so we view $G$ as a subgroup), that $t$ generates an infinite cyclic subgroup $\langle t\rangle \leq G *_{\varphi}$, and that $G \cap\langle t\rangle=\{1\}$. We refer to [Co] or [LS] for these and additional properties of HNN extensions.

A special case of HNN extension occurs when $H=H^{\prime}=G$. In that situation, the HNN extension is a semidirect product of $G$ with $\langle t\rangle$ with respect to the automorphism $\varphi: G \rightarrow G$. That group will be denoted $G \rtimes_{\varphi}\langle t\rangle$ or-when the specific isomorphism is not important-just $G \rtimes\langle t\rangle$. A nice exposition of semidirect products is in [Me, Ch.8].

Some of the topological constructions used in this paper produce entire inverse sequences of HNN extensions and/or semidirect products from an initial "base" sequence. Of special interest here is the extent to which a given property of one of those sequences implies the same property for the other. Before formulating some propositions of that sort, we state a pair of elementary observations and a pair of corollaries that will be used throughout; proofs are left as exercises.

Lemma 2.1. Let $G_{0}$ and $G_{1}$ be groups and $\varphi_{i}: H_{i} \rightarrow H_{i}^{\prime}$ an isomorphism between subgroups of $G_{i}$ for $i=0,1$. Suppose $\lambda: G_{1} \rightarrow G_{0}$ is a homomorphism taking $H_{1}$ into $H_{0}$ and $H_{1}^{\prime}$ into $H_{0}^{\prime}$ such that $\varphi_{0} \circ \lambda(h)=\lambda \circ \varphi_{1}(h)$ for all $h \in H_{1}$. Then $\lambda$ induces a unique homomorphism $\bar{\lambda}: G_{1} *_{\varphi_{1}} \rightarrow G_{0} *_{\varphi_{0}}$ that restricts to $\lambda$ on $G_{1}$ and sends the stable letter $t_{1}$ to the stable letter $t_{0}$.

Lemma 2.2. Given the above setup, the homomorphism $\varphi_{0}$ restricts to an isomorphism $\psi_{0}$ of $\lambda\left(H_{1}\right)$ onto $\lambda\left(H_{1}^{\prime}\right)$. If we let $v: \operatorname{Im}(\lambda) \hookrightarrow G_{0}$ be inclusion and $\delta: G_{1} \rightarrow \operatorname{Im}(\lambda)$ the corestriction of $\lambda$, then the induced homomorphisms provided by Lemma 2.1 yield a commutative diagram:

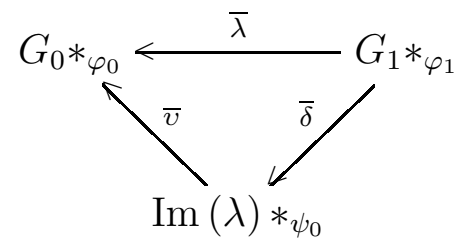

Corollary 2.3. If, under the setup of Lemma 2.1, $H_{i}=H_{i}^{\prime}=G_{i}$ for each $i$, then $\lambda$ induces a unique homomorphism $\bar{\lambda}: G_{1} \rtimes_{\varphi_{1}}\left\langle t_{1}\right\rangle \rightarrow G_{0} \rtimes_{\varphi_{0}}\left\langle t_{0}\right\rangle$ that restricts to $\lambda$ on $G_{1}$ and takes $t_{1}$ to $t_{0}$.

Corollary 2.4. Given the setup of Corollary [2.3, the homomorphism $\varphi_{0}$ restricts to an automorphism $\psi_{0}$ of $\operatorname{Im}(\lambda)$. If we let $v: \operatorname{Im}(\lambda) \hookrightarrow G_{0}$ be inclusion and $\delta: G_{1} \rightarrow \operatorname{Im}(\lambda)$ the corestriction of $\lambda$, then the induced homomorphisms provided by Corollary 2.3 yield a commutative diagram:

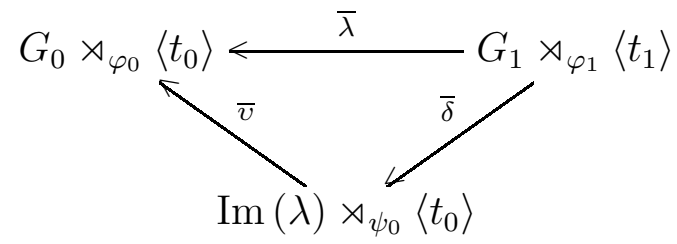

Since $\bar{v}$ is injective, we view it as an inclusion map. 
For the remainder of this section, we work with the following setup:

(a) $G_{0} \stackrel{\lambda_{1}}{\longleftarrow} G_{1} \stackrel{\lambda_{2}}{\longleftarrow} G_{2} \stackrel{\lambda_{3}}{\longleftarrow} \cdots$ is an inverse sequence of groups (the base sequence),

(b) for each $i, H_{i}, H_{i}^{\prime} \leq G_{i}$ and $\varphi_{i}: H_{i} \rightarrow H_{i}^{\prime}$ is an isomorphism, and

(c) for each $i, \varphi_{i-1} \circ \lambda_{i}(h)=\lambda_{i} \circ \varphi_{i}(h)$ for all $h \in H_{i}$.

Under these assumptions, repeated application of Lemma 2.1 gives the following induced HNN sequence:

$$
G_{0} *_{\varphi_{0}} \stackrel{\bar{\lambda}_{1}}{\longleftarrow} G_{1} *_{\varphi_{1}} \stackrel{\bar{\lambda}_{2}}{\longleftarrow} G_{2} * \varphi_{2} \stackrel{\bar{\lambda}_{3}}{\longleftarrow} \cdots
$$

If we strengthen condition (b) to:

$\left(\mathrm{b}^{\prime}\right)$ for each $i, \varphi_{i} \in \operatorname{Aut}\left(G_{i}\right)$,

then repeated application of Corollary 2.3 gives the following induced semidirect product sequence:

$$
G_{0} \rtimes_{\varphi_{0}}\left\langle t_{0}\right\rangle \stackrel{\bar{\lambda}_{1}}{\longleftarrow} G_{1} \rtimes_{\varphi_{1}}\left\langle t_{1}\right\rangle \stackrel{\bar{\lambda}_{2}}{\longleftarrow} G_{2} \rtimes_{\varphi_{2}}\left\langle t_{2}\right\rangle \stackrel{\bar{\lambda}_{3}}{\longleftarrow} \cdots
$$

The situation for semidirect products is less complicated than for general HNN extensions, so we turn to that case first.

Proposition 2.5. Assume we are given the above setup, with Condition ( $\left.b^{\prime}\right)$ in place of (b). Then

(1) The induced semidirect product sequence is pro-monomorphic if and only if the base sequence is pro-monomorphic.

(2) The induced semidirect product sequence is semistable if and only if the base sequence is semistable.

(3) The induced semidirect product sequence is stable if and only if the base sequence is stable.

Proof. By repeated application of Lemma 2.2 we may obtain a ladder diagram of the following type:

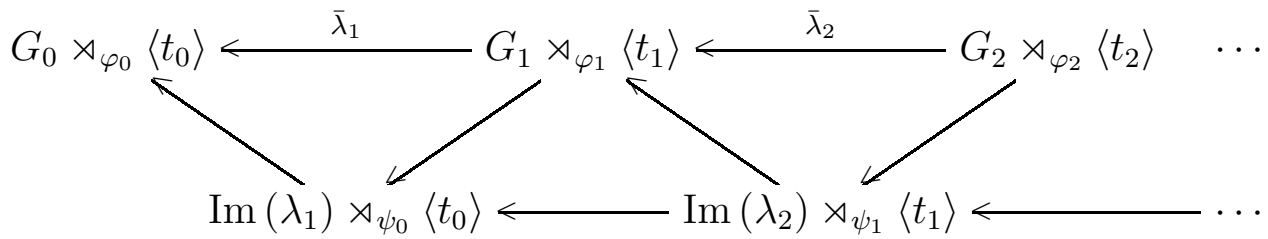

To prove (1), first assume that $\left\{G_{i}, \lambda_{i}\right\}$ is pro-monomorphic. Then, after passing to a subsequence and relabeling, we may assume that the bonding maps in the corresponding sequence $\operatorname{Im}\left(\lambda_{1}\right) \leftarrow \operatorname{Im}\left(\lambda_{2}\right) \leftarrow \operatorname{Im}\left(\lambda_{3}\right) \leftarrow \cdots$ are monomorphisms. Elementary properties of semidirect products then ensure that the maps in the bottom row of the ladder diagram are also monomorphisms. It follows that the induced semidirect product sequence is pro-monomorphic.

For the converse part of (1), notice that (even before passing to subsequences), $\operatorname{Im}\left(\bar{\lambda}_{i}\right)=\operatorname{Im}\left(\lambda_{i}\right) \rtimes_{\psi_{0}}\left\langle t_{i-1}\right\rangle \leq G_{i-1} \rtimes_{\varphi_{1}}\left\langle t_{i-1}\right\rangle$, for all $i$. So by hypothesis, after 
passing to subsequences and relabeling, we may assume the existence of a sequence of the form

$$
\operatorname{Im}\left(\lambda_{1}\right) \rtimes_{\psi_{0}}\left\langle t_{0}\right\rangle \longleftarrow \operatorname{Im}\left(\lambda_{2}\right) \rtimes_{\psi_{1}}\left\langle t_{1}\right\rangle \longleftarrow \operatorname{Im}\left(\lambda_{3}\right) \rtimes_{\psi_{2}}\left\langle t_{2}\right\rangle \longleftarrow \cdots
$$

Then each of the above monomorphisms restricts to a monomorphism of $\operatorname{Im}\left(\lambda_{i+1}\right)$ into $\operatorname{Im}\left(\lambda_{i}\right)$. It follows that $\left\{G_{i}, \lambda_{i}\right\}$ is pro-monomorphic.

A similar strategy can be used to obtain (2), with a key ingredient being that the homomorphisms $\operatorname{Im}\left(\lambda_{i}\right) \rtimes_{\psi_{i}}\left\langle t_{i}\right\rangle \rightarrow \operatorname{Im}\left(\lambda_{i-1}\right) \rtimes_{\psi_{i-1}}\left\langle t_{i-1}\right\rangle$ are surjective if and only if $\operatorname{Im}\left(\lambda_{i}\right) \rightarrow \operatorname{Im}\left(\lambda_{i-1}\right)$ is surjective.

Lastly, one may obtain (3) as a consequence of (1) and (2).

As noted earlier, the situation for general HNN extensions is more complicated. Our best analog of Proposition 2.5 is the following:

Proposition 2.6. Assume we are given Conditions (a)-(c). Then

(1) If the induced HNN sequence is pro-monomorphic, then the base sequence is pro-monomorphic.

(2) If the base sequence is semistable, then the induced HNN sequence is semistable.

Proof. First we prove (2). By hypothesis, after passing to a subsequence and relabeling, we may assume an inverse sequence of surjections:

$$
\operatorname{Im}\left(\lambda_{1}\right) \nleftarrow \operatorname{Im}\left(\lambda_{2}\right) \nleftarrow \operatorname{Im}\left(\lambda_{3}\right) \nleftarrow \cdots .
$$

By repeated application of Lemma 2.2, obtain a corresponding ladder diagram of the form:

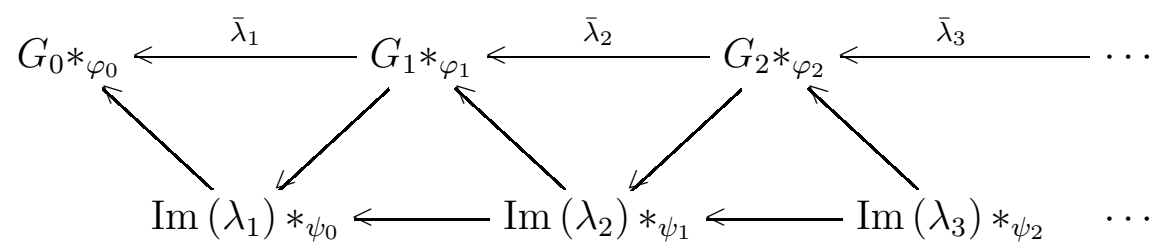

Since each $\operatorname{Im}\left(\lambda_{i+1}\right) *_{\psi_{i}}$ is generated by the elements of $\operatorname{Im}\left(\lambda_{i+1}\right)$ together with the stable letter, the homomorphisms in the bottom row are surjective. Thus, $\left\{G_{i} *_{\varphi_{i}}, \bar{\lambda}_{i}\right\}$ is semistable.

In proving (1), a key difference between this and Proposition 2.5 becomes important. The "up maps" in the current ladder diagram need not be injective, so the groups in the bottom row are not just the images of the maps in the top row (as was the case in Proposition 2.5). Instead of proceeding in the manner of the earlier proof, we give an argument based on first principles.

Suppose the base sequence $\left\{G_{i}, \lambda_{i}\right\}$ is not pro-monomorphic. Then for every $j_{1}$ there exists $j_{2}>j_{1}$ such that for arbitrarily large $j_{3}>j_{2}$ there exists a $g \in G_{j_{3}}$ such that $g \in \operatorname{ker} \lambda_{j_{3} j_{1}}$ but $g \notin \operatorname{ker} \lambda_{j_{3} j_{2}}$. Since each $\bar{\lambda}_{i}$ acts as $\lambda_{i}$ on $G_{i}<G_{i} * \varphi_{i}$, one sees the same non-pro-monomorphic behavior in $\left\{G_{i} *_{\varphi_{i}}, \bar{\lambda}_{i}\right\}$.

Proposition 2.6 may be most interesting for what is not included. The absence of a converse for assertion (2) has significant implications for this paper, as does 
the corresponding existence of a converse in Proposition 2.5. For completeness, we provide examples covering the missing implications of Proposition 2.6

Example 3. Here we describe a situation where the base sequence is stable, but the induced HNN sequence is not pro-monomorphic. Begin with the sequence

$$
\mathbb{Z} \stackrel{\text { id }}{\longleftarrow} \mathbb{Z} \stackrel{\text { id }}{\longleftarrow} \mathbb{Z} \stackrel{\text { id }}{\longleftarrow} \cdots .
$$

For the $i^{\text {th }}$ copy of $\mathbb{Z}$, let $H_{i}=H_{i}^{\prime}=2^{i} \mathbb{Z}$ and let $\varphi_{i}$ be the identity homomorphism. Then the resulting $H N N$ sequence

$$
\mathbb{Z} *_{\varphi_{1}} \stackrel{\overline{\mathrm{id}}}{\longleftarrow} \mathbb{Z} *_{\varphi_{2}} \stackrel{\overline{\mathrm{id}}}{\longleftarrow} \mathbb{Z} *_{\varphi_{3}} \stackrel{\overline{\mathrm{id}}}{\longleftarrow} \cdots
$$

consists of non-monomorphic surjections - a situation never pro-isomorphic to a sequence of monomorphisms.

Example 4. Here is a situation where a non-semistable base sequence gives rise to an induced $H N N$ sequence that is stable. Let $G_{0}=\langle a\rangle$ be an infinite cyclic group, $G_{1}=\left\langle a^{2}\right\rangle$, and $\lambda$ the inclusion map. Let $H_{0}=\left\langle a^{2}\right\rangle, H_{0}^{\prime}=\langle a\rangle$, and $\varphi_{0}:\left\langle a^{2}\right\rangle \rightarrow\langle a\rangle$ the isomorphism taking $a^{2}$ to a. Similarly, let $H_{1}=\left\langle a^{4}\right\rangle, H_{1}^{\prime}=\left\langle a^{2}\right\rangle$, and $\varphi_{1}:\left\langle a^{4}\right\rangle \rightarrow\left\langle a^{2}\right\rangle$ the isomorphism taking $a^{4}$ to $a^{2}$. Then we have presentations

$$
\begin{aligned}
& G_{0} *_{\varphi_{0}}=\left\langle a, t \mid t^{-1} a^{2} t=a\right\rangle, \text { and } \\
& G_{1} *_{\varphi_{1}}=\left\langle a^{2}, t \mid t^{-1} a^{4} t=a^{2}\right\rangle .
\end{aligned}
$$

Each is the well-known Baumslag-Solitar group BS $(2,1)$. Clearly, the induced homomorphism $\bar{\lambda}: G *_{\varphi_{1}} \rightarrow G *_{\varphi_{0}}$ takes $t$ to $t$ and $a^{2}$ to $a^{2}$. Applying Tietze transformations to the presentation for $G_{1} *_{\varphi_{1}}$ yields

$$
\begin{aligned}
\left\langle a^{2}, t \mid t^{-1} a^{4} t=a^{2}\right\rangle & \rightsquigarrow\left\langle a^{2}, t, b \mid t^{-1} a^{4} t=a^{2}, b=t^{-1} a^{2} t\right\rangle \\
& \rightsquigarrow\left\langle a^{2}, t, b \mid b^{2}=t b t^{-1}, t b t^{-1}=a^{2}\right\rangle \\
& \rightsquigarrow\left\langle t, b \mid b^{2}=t b t^{-1}\right\rangle
\end{aligned}
$$

With respect to the final presentation, $\bar{\lambda}$ takes $t$ to $t$ and $b$ to $t^{-1} a^{2} t=a$. Thus, $\bar{\lambda}$ is an isomorphism.

By applying the above observation inductively, we can begin with the pro-monomorphic but non-semistable sequence

$$
\langle a\rangle \hookleftarrow\left\langle a^{2}\right\rangle \hookleftarrow\left\langle a^{4}\right\rangle \hookleftarrow\left\langle a^{8}\right\rangle \hookleftarrow \cdots
$$

and end up with a corresponding HNN sequence

$$
\left\langle a, t \mid t^{-1} a^{2} t=a\right\rangle \leftarrow\left\langle a^{2}, t \mid t^{-1} a^{4} t=a^{2}\right\rangle \leftarrow\left\langle a^{4}, t \mid t^{-1} a^{8} t=a^{4}\right\rangle \leftarrow \cdots
$$

of isomorphisms between copies of $B S(2,1)$.

We conclude this section with an observation covering a very special case of an induced HNN sequence. It will be used in $₫ 9$. 
Lemma 2.7. Suppose that, in addition to conditions (a)-(c), the inverse sequences: $G_{0} \stackrel{\lambda_{1}}{\longleftarrow} G_{1} \stackrel{\lambda_{2}}{\longleftarrow} G_{2} \stackrel{\lambda_{3}}{\longleftarrow} \cdots$ and $H_{0} \stackrel{\mu_{1}}{\longleftarrow} H_{1} \stackrel{\mu_{2}}{\longleftarrow} H_{2} \stackrel{\mu_{3}}{\longleftarrow} \cdots$ (and hence $H_{0}^{\prime} \stackrel{\mu_{1}^{\prime}}{\longleftarrow}$ $H_{1}^{\prime} \stackrel{\mu_{2}^{\prime}}{\longleftarrow} H_{2}^{\prime} \stackrel{\mu_{3}^{\prime}}{\longleftarrow}$...) are stable, where $\mu_{i}$ and $\mu_{i}^{\prime}$ denote appropriate restrictions of $\lambda_{i}$ Then $\underline{\varphi}=\left(\varphi_{i}\right)_{i \geq 0}$ defines an isomorphism between subgroups $\underline{H}=\lim \left\{H_{i}, \mu_{i}\right\}$ and $\underline{H}^{\prime}=\lim _{\longleftarrow}\left\{H_{i}^{\prime}, \mu_{i}^{\prime}\right\}$ of $\underline{G}=\lim _{\longleftarrow}\left\{G_{i}, \lambda_{i}\right\}$; moreover, the induced HNN sequence $G_{0} *_{\varphi_{0}} \stackrel{\bar{\lambda}_{1}}{\longleftarrow} G_{1} *_{\varphi_{1}} \stackrel{\bar{\lambda}_{2}}{\longleftarrow} G_{2} *_{\varphi_{2}} \stackrel{\bar{\lambda}_{3}}{\longleftarrow} \cdots$ is stable and pro-isomorphic to the constant sequence $\{\underline{G} * \underline{\varphi}\}$.

Proof. By the stability if $\left\{G_{i}, \lambda_{i}\right\}$ we may, after passing to a subsequence and relabeling, assume that

$$
\operatorname{Im}\left(\lambda_{1}\right) \cong \operatorname{Im}\left(\lambda_{2}\right) \cong \operatorname{Im}\left(\lambda_{3}\right) \cong \ldots
$$

By passing to a further subsequence and relabeling again, we may assume that, within each $\operatorname{Im}\left(\lambda_{i}\right)$ lies the subgroup $\lambda_{i+1}\left(H_{i+1}\right)$, and the restriction of $\lambda_{i+1}$ takes $\lambda_{i+2}\left(H_{i+2}\right)$ isomorphically onto $\lambda_{i+1}\left(H_{i+1}\right)$ for each $i$. The analogous conditions for the primed subgroups follow automatically. Passing to the corresponding HNN sequence for this system yields an inverse sequence of canonical isomorphisms between groups of the form $\operatorname{Im}\left(\lambda_{i+1}\right) *_{\psi_{i}}$ where $\psi_{i} \mid$ takes $\lambda_{i+1}\left(H_{i+1}\right)$ isomorphically onto $\lambda_{i+1}\left(H_{i+1}^{\prime}\right)$. The projection maps are then isomorphisms from $\underline{G} *_{\underline{\varphi}}$ to $\operatorname{Im}\left(\lambda_{i+1}\right) *_{\psi_{i}}$.

Remark 5. Example 4 shows that, in general, we cannot expect $\lim _{\bar{\lambda}}\left\{G_{i} *_{\varphi_{i}}, \bar{\lambda}_{i}\right\}$ to be isomorphic to $\lim _{\longleftarrow}\left\{G_{i}, \lambda_{i}\right\} *_{\varphi}$. In that particular case, $\lim _{\longleftarrow}\left\{G_{i} *_{\varphi_{i}}, \bar{\lambda}_{i}\right\}$ is isomorphic to $B S(2,1)$ while $\lim _{\longleftarrow}\left\{G_{i}, \lambda_{i}\right\} *_{\underline{\varphi}} \cong \mathbb{Z}$, since $\lim _{\longleftarrow}\left\{G_{i}, \lambda_{i}\right\}$ is the trivial group.

2.2. Topology at the end of a space. In this paper, all spaces are assumed to be connected, locally compact, separable, and metrizable. A space $X$ is an $A N R$ (absolute neighborhood retract) if, whenever it is embedded as a closed subset of a metric space $Z$, some neighborhood $U$ of $X$ retracts onto $X$. It is well-known that manifolds and locally finite CW complexes are ANRs. While many readers will want to focus their attention on manifolds and CW complexes, results presented here are valid for all ANRs satisfying the stated topological assumptions. Portions of this paper, such as Proposition 7.1, are valid for even more general spaces

A subset $N$ of a space $X$ is a neighborhood of infinity if $\overline{X-N}$ is compact. Standard arguments show that, when $X$ satisfies the conditions in the previous paragraph, and $C$ is a compact subset of $X$, then $X-C$ contains at most finitely many unbounded components, i.e., finitely many components with noncompact closures. If $X-C$ has both bounded and unbounded components, the situation can be simplified by letting $C^{\prime}$ consist of $C$ together with all bounded components. Then $C^{\prime}$ is compact, and $X-C^{\prime}$ consists entirely of unbounded components.

We say that $X$ has $k$ ends if there exists a compactum $C \subseteq X$ such that, for every compactum $D$ with $C \subset D, X-D$ has exactly $k$ unbounded components. When $k$ exists, it is uniquely determined; if $k$ does not exist, we say $X$ has infinitely many 
ends. Thus, a space is 0-ended if and only if $X$ is compact, and 1-ended if and only if it contains arbitrarily small connected neighborhoods of infinity.

A nested sequence $N_{0} \supseteq N_{1} \supseteq N_{2} \supseteq \cdots$ of neighborhoods of infinity, with each $N_{i} \subseteq \operatorname{int} N_{i-1}$, is cofinal if $\bigcap_{i=0}^{\infty} N_{i}=\varnothing$. Such a sequence is easily obtained: choose an exhaustion of $X$ by compacta $C_{0} \subseteq C_{1} \subseteq C_{2} \subseteq \cdots$, with each $C_{i-1} \subseteq \operatorname{int} C_{i}$; then let $N_{i}=X-C_{i}$.

Given a nested cofinal sequence $\left\{N_{i}\right\}_{i=0}^{\infty}$ of connected neighborhoods of infinity, base points $p_{i} \in N_{i}$, and paths $r_{i} \subset N_{i}$ connecting $p_{i}$ to $p_{i+1}$, we obtain an inverse sequence:

$$
\pi_{1}\left(N_{0}, p_{0}\right) \stackrel{\lambda_{1}}{\longleftarrow} \pi_{1}\left(N_{1}, p_{1}\right) \stackrel{\lambda_{2}}{\longleftarrow} \pi_{1}\left(N_{2}, p_{2}\right) \stackrel{\lambda_{3}}{\longleftarrow} \cdots
$$

Here, each $\lambda_{i+1}: \pi_{1}\left(N_{i+1}, p_{i+1}\right) \rightarrow \pi_{1}\left(N_{i}, p_{i}\right)$ is the homomorphism induced by inclusion followed by the change of base point isomorphism determined by $r_{i}$. The proper ray $r:[0, \infty) \rightarrow X$ obtained by piecing together the $r_{i}$ 's in the obvious manner is referred to as the base ray for the inverse sequence, and the pro-isomorphism class of the inverse sequence is called the fundamental pro-group of $X$ based at $r$ and is denoted pro- $\pi_{1}(X, r)$. It is a standard fact that pro- $\pi_{1}(X, r)$ is independent of the cofinal sequence of neighborhoods $\left\{N_{i}\right\}$ or the base points-provided those base points tend to infinity along the ray $r$. The pro-isomorphism class is also independent of the parameterization of $r$ and, more generally, is independent of the proper homotopy class within $X$ of $r$. At times we take the inverse limit of pro- $\pi_{1}(X, r)$; the result is called the $\check{C}$ ech fundamental group of $X$ based at $r$ and is denoted by $\check{\pi}_{1}(X, r)$.

Clearly, if $X$ has more than one end, the "choice of ends to which $r$ points" will affect pro- $\pi_{1}(X, r)$ (and thus, $\check{\pi}_{1}(X, r)$ ). On a more subtle note, even if $X$ has a single end, pro- $\pi_{1}$ may not be independent of base ray. For the purposes of this paper, this issue causes no problems - all concerns can be addressed by a pair of standard propositions given below. Both can be found in $\mathrm{Ge}$ along with a more thorough discussion of the the non-uniqueness issue for pro- $\pi_{1}$.

For simplicity, the following are formulated only for the case where $X$ is one-ended.

Proposition 2.8. Let $X$ be a one-ended space and $r:[0, \infty) \rightarrow X$ a proper ray. Then the following are equivalent:

(1) $\operatorname{pro}-\pi_{1}(X, r)$ is semistable.

(2) All proper rays in $X$ are properly homotopic.

Proposition 2.9. Let $X$ be a one-ended space and $r:[0, \infty) \rightarrow X$ a proper map. Then the following are equivalent:

(1) $\operatorname{pro}_{1}(X, r)$ is pro-monomorphic.

(2) There exists a compact $C \subseteq X$ such that, for every compact set $D$ containing $C$, there exists a compact $E$ such that every loop in $X-E$ that contracts in $X-C$ contracts in $X-D$.

A compactum $C \subseteq X$ with the property described in Proposition [2.9 is called a compact core; thus, the proposition may be restated to say: pro- $\pi_{1}(X, r)$ is promonomorphic if and only if $X$ contains a compact core. 
Since neither condition (2) in the above two propositions involves a base ray, it follows that (in the one-ended case) having a pro-monomorphic or semistable pro$\pi_{1}(X, r)$ for some $r$ is equivalent to having pro-monomorphic or semistable pro- $\pi_{1}$ for all base rays. For that reason, we simply say " $X$ has pro-monomorphic [resp., semistable] fundamental group at infinity".

Remark 6. Proposition 2.8 provides even more. In the presence of semistability, the fundamental group at infinity of a one-ended space is well-defined up to proisomorphism - this is analogous to the fact that the fundamental group of a pathconnected space is well-defined up to isomorphism. In the pro-monomorphic situation this is not always the case.

As a final preliminary, we discuss the manner in which a ladder diagram of groups of type (2.1) arises in the study of fundamental pro-groups. In doing so, we highlight the need for passage to subsequences and address some issues related to base points and base rays. For simplicity, consider the case of a homeomorphism $h: P \rightarrow Q$ between noncompact spaces. For a proper ray $r:[0, \infty) \rightarrow P$, we will exhibit the equivalence of pro- $\pi_{1}(P, r)$ and pro- $\pi_{1}(Q, h \circ r)$. Rather that opting for the most concise treatment, we give an approach that closely resembles the situation that arises in the proof of our main theorem.

Let $\left\{U_{i}\right\}$ and $\left\{V_{i}\right\}$ be cofinal sequences of neighborhoods of infinity in $P$ and $Q$, respectively. By discarding an initial segment of the base ray and reparameterizing, we may assume that $r([i, \infty)) \subseteq U_{i}$ for all integers $i \geq 0$. Next we choose "interlocking" subsequences of $\left\{U_{i}\right\}$ and $\left\{V_{i}\right\}$. Let $V_{j_{0}}=V_{0}$, then choose $k_{0}$ sufficiently large that $h\left(U_{k_{0}}\right) \subseteq V_{j_{0}}$; this is possible since homeomorphisms are proper functions. Next choose $j_{1}>j_{0}$ large enough so that $h^{-1}\left(V_{j_{1}}\right) \subseteq U_{k_{0}}$; then choose $k_{1}>k_{0}$ so that $h\left(U_{k_{1}}\right) \subseteq V_{j_{1}}$. By continuing this process inductively, one obtains a pair of subsequences and a commutative ladder diagram

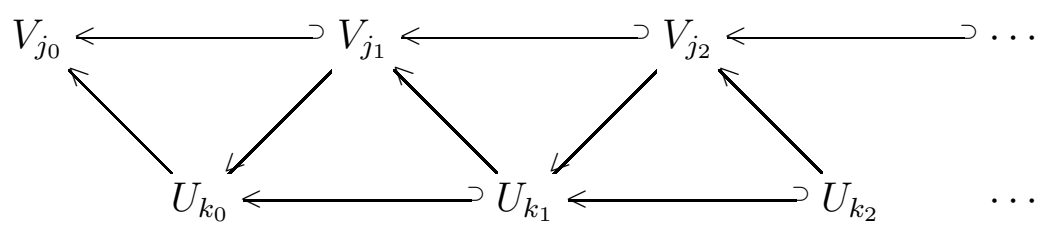

where each "up arrow" is a restriction of $h$ and each "down arrow" is a restriction of $h^{-1}$.

We wish to apply the fundamental group functor to the above setup to get a diagram of the form

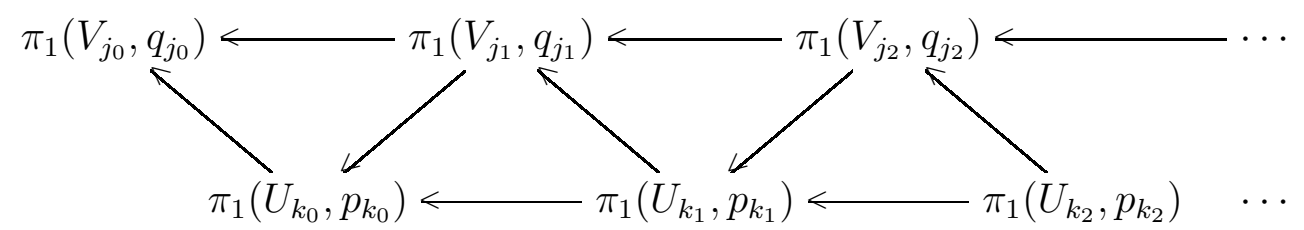

This will require choices of base points and base rays. In order to define the up and down homomorphisms resulting in a commutative diagram, those choices must be 
made in a consistent manner. The first key choice was in selecting $h \circ r$ as the base ray for $Q$. Since $r\left(\left[k_{i}, \infty\right)\right) \subseteq U_{k_{i}}$ for all $i$, then $h \circ r\left(\left[k_{i}, \infty\right)\right) \subseteq V_{j_{i}}$. We will not take the trouble of reparameterizing $h \circ r$; instead for each $i \geq 0$, let $p_{k_{i}}=r\left(k_{i}\right)$ and $q_{j_{i}}=h\left(p_{k_{i}}\right)$ be the preferred base points for $U_{k_{i}}$ and $V_{j_{i-1}}$. The horizontal bonding homomorphisms have already been discussed; restrictions of $h$ induce the desired up homomorphisms $\pi_{1}\left(U_{k_{i}}, p_{k_{i}}\right) \rightarrow \pi_{1}\left(V_{j_{i}}, q_{j_{i}}\right)$. Meanwhile, restrictions of $h^{-1}$ induce homomorphism $\pi_{1}\left(V_{j_{i}}, q_{j_{i}}\right) \rightarrow \pi_{1}\left(U_{k_{i-1}}, p_{k_{i}}\right)$. To get the appropriate "down homomorphism" these are composed with change-of-base point isomorphisms $\pi_{1}\left(U_{k_{i-1}}, p_{k_{i}}\right) \rightarrow \pi_{1}\left(U_{k_{i-1}}, p_{k_{i-1}}\right)$ induced by the paths $\left.r\right|_{\left[k_{i-1}, k_{i}\right]}$. Since bonding homomorphisms in the bottom row were also defined using change-of-base point isomorphisms with respect to the same set of paths, triangles of homomorphisms with a vertex on the top row are commutative. To see that triangles with a vertex on the bottom row also commute, it suffices to follow the image of a single loop - the key is that the change-of-base point path in $P$ used to define the down homomorphism is taken by $h$ to the change-of-base point path in $Q$ used to define the horizontal bonding homomorphism.

\section{RECTANGULAR NEIGHBORHOODS OF INFINITY AND THEIR FUNDAMENTAL GROUPS}

In this section we focus on spaces of the form $Y \times \mathbb{R}$, where $Y$ is connected and noncompact.

Since $Y \times \mathbb{R}$ contains arbitrarily large compacta of the form $K \times[u, v]$, it contains arbitrarily small neighborhoods of infinity of the form $\overline{(Y \times \mathbb{R})-(K \times[u, v])}$. Such a neighborhood of infinity will be called rectangular and denoted $R(K \times[u, v])$. Specifically,

$$
R(K \times[u, v])=\overline{(Y \times \mathbb{R})-(K \times[u, v])}=(\overline{Y-K} \times \mathbb{R}) \cup(Y \times(-\infty, u] \cup[v, \infty)) .
$$

It is easy to see that rectangular neighborhoods of infinity are connected and, by the Generalized Seifert-VanKampen Theorem (see, for example, [Ge, Th. 6.2.11]), the fundamental group of $R(K \times[u, v])$ is isomorphic to the fundamental group of the following graph of groups, were the vertex groups $\pi_{1}(Y)$ correspond to $\pi_{1}(Y \times(-\infty, u])$ and $\pi_{1}(Y \times[v, \infty))$ and the edges correspond to the components $U_{i}$ of $Y-K$ with $\Lambda_{i}=\operatorname{Im}\left(\pi_{1}\left(\bar{U}_{i}\right) \rightarrow \pi_{1}(Y)\right)$; the homomorphisms of the $\Lambda_{i}$ into the vertex groups are induced by inclusions.

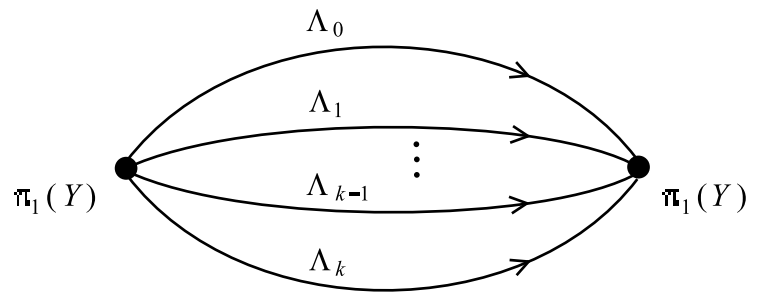

In this paper, we are particularly interested in spaces of the form $(J \backslash X) \times \mathbb{R}$, where $X$ is a one-ended simply connected space and $J$ is an infinite cyclic group acting on $X$ by covering transformations. In that case, the vertex groups will be isomorphic 
to $\mathbb{Z}$ and each edge group may be viewed as $n_{i} \mathbb{Z}$ for some integer $n_{i}$. Furthermore, by choosing $K$ so that $(J \backslash X)-K$ has only unbounded components, we can ensure that each $n_{i}$ is nonzero. Indeed, if some $n_{i}$ were equal to 0 , then the corresponding unbounded component $U_{i}$ of $(J \backslash X)-K$ would have infinitely many homeomorphic preimages under the covering projection $X \rightarrow J \backslash X$. Since $U_{i}$ has compact boundary, that would violate the one-endedness of $X$. If $k+1$ is the number of components of $(J \backslash X)-K$, the resulting graph of groups

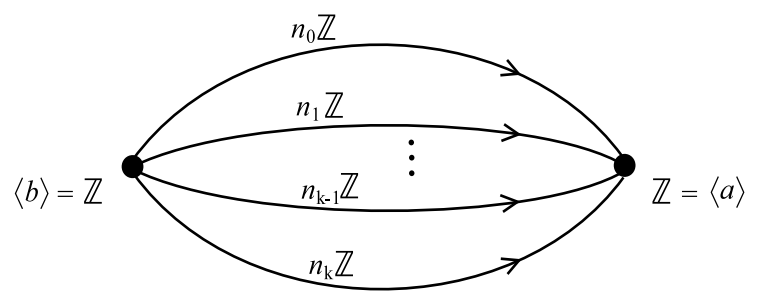

has a fundamental group $\Theta$ with presentation

$$
\left.\Theta=\left\langle a, b, s_{1}, \cdots, s_{k}\right| a^{n_{0}}=b^{n_{0}}, a^{n_{i}}=s_{i} b^{n_{i}} s_{i}^{-1} \text { for } i=1, \cdots, k\right\rangle .
$$

In applying the Generalized Seifert-VanKampen Theorem to obtain this presentation, numerous choices of base points, base paths, and a base tree must be made. All of that is set out carefully in [GM]. We provide a brief description and refer the reader to GM for precise details.

For a given base point $p_{0} \in J \backslash X$, the letters $a$ and $b$ represent corresponding generators of $\pi_{1}\left((J \backslash X) \times\{v+1\},\left(p_{0}, v+1\right)\right)$ and $\pi_{1}\left((J \backslash X) \times\{u-1\},\left(p_{0}, u-1\right)\right)$ (the latter connected to $\left(p_{0}, v+1\right)$ by a base path), respectively. If $U_{0}, U_{1}, \cdots U_{k}$ are the components of $(J \backslash X)-K$, with a base point $z_{i} \in U_{i}$ for each $i$; then the generator $s_{i}$ corresponds to a loop that passes from $\left(p_{0}, v+1\right)$ to $\left(z_{i}, v+1\right)$ in $(J \backslash X) \times\{v+1\}$ then travels down the interval $z_{i} \times[u-1, v+1]$ to $\left(z_{i}, u-1\right)$ before moving within $(J \backslash X) \times\{u-1\}$ to $\left(z_{0}, u-1\right)$, then up the interval $z_{0} \times[u-1, v+1]$ before returning to $\left(p_{0}, v+1\right)$. For consistency, all base points and all non-specified paths are required to have first coordinate a prechosen tree $T \subseteq J \backslash X$. For convenience, we will refer $a$ and $b$ as the primary generators of $\Theta$ and $s_{1}, \cdots, s_{k}$ as the secondary generators. A key algebraic fact presented in [GM] is that $\Theta$ has a nontrivial center generated by $a^{N}\left(=b^{N}\right)$, where $N$ is the least common multiple of $\left\{n_{0}, n_{1}, \cdots, n_{k}\right\}$.

Given a compactum $K^{\prime} \supseteq K$ and integers $u^{\prime}<u<v<v^{\prime}$, we now look at the inclusion-induced homomorphism between the fundamental groups of $R\left(K^{\prime} \times\left[u^{\prime}, v^{\prime}\right]\right)$ and $R(K \times[u, v])$. Choose $K^{\prime}$ so that components $U_{1}^{\prime}, \cdots, U_{k^{\prime}}^{\prime}$ of $(J \backslash X)-K^{\prime}$ are all unbounded, and note that whenever $U_{j}^{\prime} \subseteq U_{i}$ then $n_{i}$ divides $n_{j}^{\prime}$; By choosing base points, base paths, and base trees that are parallel to those used for $R(K \times[u, v])$ (in a sense made precise in [GM] $)$ we obtain a similar presentation for the fundamental group $\Theta^{\prime}$ of $R\left(K^{\prime} \times\left[u^{\prime}, v^{\prime}\right]\right)$ based at $\left(p_{0}, v^{\prime}+1\right)$ :

$$
\left.\Theta^{\prime}=\left\langle a, b, s_{1}^{\prime}, \cdots, s_{k^{\prime}}^{\prime}\right| a^{n_{0}^{\prime}}=b^{n_{0}^{\prime}}, a^{n_{i}^{\prime}}=s_{i}^{\prime} b^{n_{i}^{\prime}} s_{i}^{\prime-1} \text { for } i=1, \cdots, k^{\prime}\right\rangle
$$


Here we abuse notation slightly by again denoting the primary generators by $a$ and $b$. This is justified by the fact that, in $R(K \times[u, v])$ the loops representing principal generators of $\pi_{1}\left(R\left(K^{\prime} \times\left[u^{\prime}, v^{\prime}\right]\right)\right)$ are parallel copies (with respect to the product structure) of those representing principal generators of $\pi_{1}(R(K \times[u, v]))$. By choosing the obvious path between base points, namely $\left\{p_{0} \times\left[v+1, v^{\prime}+1\right]\right\}$, the homomorphism $\eta: \Theta^{\prime} \rightarrow \Theta$ induced by inclusion followed by a change-of-base points isomorphism takes principal generators to principal generators. By our notational convention, we have $\eta(a)=a$ and $\eta(b)=b$. On the secondary generators, $\eta\left(s_{j}^{\prime}\right)=s_{i}$ whenever $U_{j}^{\prime} \subseteq U_{i}$. Note that, since each $U_{i}$ contains at least one component of $(J \backslash X)-K^{\prime}, \eta$ is surjective.

Now choose an exhausting sequence $K_{0} \subseteq K_{1} \subseteq K_{2} \subseteq$. of compacta in $J \backslash X$ with each $K_{i} \subseteq \operatorname{int} K_{i+1}$ and each $(J \backslash X)-K_{i}$ having only unbounded components. If we also choose a monotone sequence $\left\{v_{i}\right\}$ of positive integers approaching $\infty$ and a monotone sequence $\left\{u_{i}\right\}$ of negative integers approaching $-\infty$ along with base points, base paths and base trees as described above, then we obtain an inverse sequence representing pro- $\pi_{1}((J \backslash X) \times \mathbb{R}, r)$ for which all groups are of the type described in (3.1) and (3.2) and all bonding homomorphisms are of the type just described. In this setup, the base ray $r:[0, \infty) \rightarrow(J \backslash X) \times \mathbb{R}$ is the one obtained by piecing together the change of base point paths; therefore, it is a parameterization of $\left\{p_{0}\right\} \times\left[v_{0}+1, \infty\right)$. In a later application, it will be convenient to use a different base ray. Like the one just described, the new ray will pass through the base points $\left(p_{0}, v_{i}+1\right)$ in order and will lie entirely in $(J \backslash X) \times\left[v_{0}, \infty\right)$; but as it progresses upward with respect to the $\mathbb{R}$ component, its $J \backslash X$ component will wind once around the loop $a$ for each unit it moves upward. For that reason, the bonding homomorphism $\xi_{i+1}$ : $\Theta_{i+1} \rightarrow \Theta_{i}$ with respect to the new ray will be the conjugate by $a^{v_{i+1}-v_{i}}$ of the homomorphism described above. In this scenario, the homomorphisms become less canonical (for example, except for $a$, primary and secondary generators need not be sent to primary and secondary generators). For similar choices of base ray $r$ (connecting the chosen base points and lying entirely in $(J \backslash X) \times\left[v_{0}, \infty\right)$ ), variations in the bonding homomorphisms occur - in particular, conjugation by various powers of $a$. In all cases, the properties needed later in this paper are preserved. For easy reference, we assemble those in the following general proposition.

Proposition 3.1. Given $p_{0} \in J \backslash X$, sequences $\left\{K_{i}\right\},\left\{u_{i}\right\}$ and $\left\{v_{i}\right\}$ as described above, and base ray $r$ connecting the base points $\left(p_{0}, v_{i}+1\right)$ and lying entirely in $(J \backslash X) \times\left[v_{0}, \infty\right)$, the corresponding cofinal sequence $\left\{R\left(K_{i} \times\left[u_{i}, v_{i}\right]\right)\right\}$ of neighborhoods of infinity in $(J \backslash X) \times \mathbb{R}$ gives rise to a representative of pro- $\pi_{1}((J \backslash X) \times \mathbb{R}, r)$ of the form

$$
\Theta_{0} \stackrel{\rho_{1}}{\longleftarrow} \Theta_{1} \stackrel{\rho_{2}}{\longleftarrow} \Theta_{2} \stackrel{\rho_{3}}{\longleftarrow} \ldots
$$

where each $\Theta_{i}$ contains a nontrivial distinguished element a such that

(1) $\rho_{i}(a)=a$ for all $i$, and

(2) there exists a monotone sequence of positive integers $\left\{N_{i}\right\}$ such that, for each $i, a^{N_{i}}$ lies in the center of $\Theta_{i}$. 
Remark 7. Note that any subsequence of 3.3 will also satisfy the properties identified in this proposition.

\section{MAPPING TORUS NEIGHBORHOODS OF INFINITY AND THEIR FUNDAMENTAL GROUPS}

Let $X$ be a space and $j: X \rightarrow X$ a map. The mapping torus of $j$ is the quotient space

$$
T_{j}(X)=X \times[0,1] /\{(x, 1) \sim(j(x), 0) \text { for all } x \in X\} .
$$

In other words, $T_{j}(X)$ is obtained from the mapping cylinder of $j$ by identifying the domain and range ends of the cylinder. In this paper, $j$ will always be a selfhomeomorphism of $X$.

For all $s \in[0,1], X \times\{s\} \hookrightarrow T_{j}(X)$ is an embedding. Amongst these "copies" of $X$ in $T_{j}(X)$, we give special designation to the image of $X \times\{0\}$. Through this embedding, we view $X$ as a subspace of $T_{j}(X)$, and for $x \in X$ and $A \subseteq X$ we have $x \in T_{j}(X)$ and $A \subseteq T_{j}(X)$.

If $U$ and $V$ are subsets of $X$ with $V \subseteq U$ and $j(V) \subseteq U$ (thus $V \subseteq j^{-1}(U)$ ), we may define the following subspace of $T_{j}(X)$ :

$$
T_{j}(U, V)=(U \times\{0\}) \cup(V \times[0,1]) / \sim
$$

where $\sim$ is the restriction of equivalence relation in (4.1). If $U$ and $V$ are neighborhoods of infinity in $X$, then $T_{j}(U, V)$ is a neighborhood of infinity in $T_{j}(X)$ which we refer to as a torus-like neighborhood of infinity. Such neighborhoods of infinity can be made arbitrarily small by choosing $U$ to be sufficiently small in $X$. The following proposition and its corollary give a nice description of the fundamental group of $T_{j}(U, V)$ in terms of the fundamental groups of $U$ and $V$. That description is valid only when $X$ is one-ended with pro-monomorphic fundamental group at infinity - a fact which explains the presence of those hypotheses in most of our results.

Proposition 4.1. Let $X$ be connected and one-ended with pro-monomorphic fundamental group at infinity. Suppose $U$ is a connected neighborhood of infinity such that $X-U$ contains a compact core, and $j: X \rightarrow X$ is a homeomorphism. Then, for sufficiently small connected neighborhoods of infinity $V \subseteq U$ and base point $p \in V$ :

(1) $j(V) \subseteq U$, and

(2) $\operatorname{ker}\left(i_{\#}\right)=\operatorname{ker}\left(\left.j\right|_{\#}\right)$ where $i_{\#}: \pi_{1}(V, p) \rightarrow \pi_{1}(U, p)$ and $\left.j\right|_{\#}: \pi_{1}(V, p) \rightarrow$ $\pi_{1}(U, j(p))$ are induced by inclusion and by the restriction of $j$, respectively.

Proof. Begin by choosing a neighborhood of infinity $U_{1}$ so small that $U_{1}, j\left(U_{1}\right)$ and $j^{-1}\left(U_{1}\right)$ are all contained in $U$. This is easy - choose $U_{1} \subseteq X-\left(j(A) \cup A \cup j^{-1}(A)\right)$ where $A=\overline{X-U}$. Applying Proposition 2.9, choose a smaller neighborhood of infinity $U_{2}$ with the property that loops in $U_{2}$ which contract in $U$ also contract in $U_{1}$. Finally, choose $V$ to be any connected neighborhood of infinity sufficiently small that both $V$ and $j(V)$ are contained in $U_{2}$. 
Let $\beta$ be a loop in $V$ based at $p$. If $\beta$ contracts in $U$, then $\beta$ also contracts in $U_{1}$; and since $j\left(U_{1}\right) \subseteq U$, then $j \circ \beta$ contracts in $U$. Therefore $\operatorname{ker}\left(i_{\#}\right) \subseteq \operatorname{ker}\left(\left.j\right|_{\#}\right)$. Conversely if $j \circ \beta$ contracts in $U$ then, since $\operatorname{Im}(j \circ \beta)$ lies in $U_{2}$, it also contracts in $U_{1}$. But $j^{-1}\left(U_{1}\right) \subseteq U$, so $\beta$ also contracts in $U$. Hence, $\operatorname{ker}\left(\left.j\right|_{\#}\right) \subseteq \operatorname{ker}\left(i_{\#}\right)$.

Corollary 4.2. Using the same hypotheses and notation as above, assume $V$ is chosen to satisfy the conclusion of Proposition 4.1. Let $\alpha$ be a path in $U$ from $p$ to $j(p)$ and $\widehat{\alpha}$ : $\pi_{1}(U, j(p)) \rightarrow \pi_{1}(U, p)$ be the corresponding change-of-base point isomorphism. Then $\pi_{1}\left(T_{j}(U, V), p\right)$ is an $H N N$ extension of $\pi_{1}(U, p)$ by $\phi$ with associated subgroups $H=$ $i_{\#}\left(\pi_{1}(V, p)\right) \cong \pi_{1}(V, p) / \operatorname{ker}\left(i_{\#}\right)$ and $H^{\prime}=\hat{\alpha}\left(\left.j\right|_{\#}\left(\pi_{1}(V, p)\right)\right.$, where the isomorphism $\phi: H \rightarrow H^{\prime}$ is induced by $\left.\hat{\alpha} \circ j\right|_{\#}$ via an application of Proposition 4.1 .

Proof. It is an immediate consequence of the Generalized Seifert-VanKampen Theorem that $\pi_{1}\left(T_{j}(U, V), p\right)$ has the form

$$
\left\langle\pi_{1}(U, p), t\right| t^{-1}\left(i_{\#}(g)\right) t\left(\hat{\alpha}\left(\left.j\right|_{\#}(g)\right)^{-1}=1 \forall g \in \pi_{1}(V, p)\right\rangle .
$$

Quotienting out by $\operatorname{ker}\left(i_{\#}\right)$ and $\operatorname{ker}\left(\left.j\right|_{\#}\right)$ we obtain a presentation

$$
\left\langle\pi_{1}(U, p), t \mid t^{-1} h t \phi(h)^{-1}=1 \forall h \in H\right\rangle \text {. }
$$

Thus,

$$
\pi_{1}\left(T_{j}(U, V), p\right) \cong\left\langle\pi_{1}(U, p), t \mid t^{-1} h t=\phi(h) \forall h \in H\right\rangle=\pi_{1}(U, p) *_{\phi} .
$$

Remark 8. For the coming applications, it is necessary to understand some specifics of the isomorphism $\pi_{1}\left(T_{j}(U, V), p\right) \stackrel{\cong}{\longrightarrow} \pi_{1}(U, p) *_{\phi}$ just obtained. Clearly, elements of $\pi_{1}(U, p) \leq \pi_{1}(U, p) *_{\phi}$ are paired with their natural preimages via the inclusion $(U, p) \hookrightarrow\left(T_{j}(U, V), p\right)$; but the remainder of the isomorphism is not canonical-it depends on our choice of the path $\alpha$ from $p$ to $j(p)$. Note that a different choice $\beta$ may alter the subgroup $H^{\prime}$, replacing it with $g H^{\prime} g^{-1}$ (and $\phi$ by a corresponding conjugate) where $g \in \pi_{1}(U, p)$ is represented by the loop $\beta * \alpha^{-1}$. More significantly, with $\alpha$ as the chosen path, a loop representing the preimage of the stable letter $t$ is obtained by following the fiber $\{p\} \times[0,1]$ of $T_{j}(X)$ from its initial point $p$ to its endpoint $j(p)$ then returning to $p$ along $\alpha^{-1}$; we denote this loop $\tau_{p, \alpha}$. If $\beta$ is used in place of $\alpha$, the new preimage of $t$ is represented by $\tau_{p, \alpha} * g^{-1}$. For clarity, let $\chi_{\alpha}$ denote the specific isomorphism

$$
\chi_{\alpha}: \pi_{1}\left(T_{j}(U, V), p\right) \stackrel{\cong}{\longrightarrow} \pi_{1}(U, p) *_{\phi}
$$

obtained when the path $\alpha$ is used. The key facts here are that $\chi_{\alpha}$ is the identity on $\pi_{1}(U, p)$ (which, as a result of Corollary 4.2, may be viewed as a subgroup of $\left.\pi_{1}\left(T_{j}(U, V), p\right)\right)$ and takes $\tau_{p, \alpha}$ to the stable letter $t$.

Proposition 4.3. For $X$ a connected, one-ended ANR with pro-monomorphic fundamental group at infinity, let 
- $\left\{U_{n}\right\}_{n=0}^{\infty}$ be a nested cofinal sequence of connected neighborhoods of infinity such that each pair $\left(U_{n}, U_{n+1}\right)$ satisfies the conditions on $(U, V)$ in Proposition 4.1.

- $r:[0, \infty) \rightarrow X$ be a proper ray such that $r([n, \infty)) \subseteq U_{n+1}$ for all $n$,

- for each $n$, let $p_{n}=r(n)$ and $r_{n}$ be the path from $p_{n}$ to $p_{n+1}$ obtained by restricting $r$ to $[n, n+1]$

- for convenience, let $G_{n}$ denote $\pi_{1}\left(U_{n}, p_{n}\right)$, and let $\lambda_{n+1}: G_{n+1} \rightarrow G_{n}$ be the homomorphism $\widehat{r}_{n} \circ i_{n+1 \#}$ where $i_{n+1}:\left(U_{n+1}, p_{n+1}\right) \hookrightarrow\left(U_{n}, p_{n+1}\right)$ and $\widehat{r}_{n}$ is the change-of-base points isomorphism corresponding to $r_{n}$.

Then pro- $\pi_{1}(X, r)$ may be represented by the inverse sequence

$$
G_{0} \stackrel{\lambda_{1}}{\longleftarrow} G_{1} \stackrel{\lambda_{2}}{\longleftarrow} G_{2} \stackrel{\lambda_{3}}{\longleftarrow} \cdots
$$

Given all of the above and a self-homeomorphism $j: X \rightarrow X$, consider the sequence $\left\{T_{j}\left(U_{n}, U_{n+1}\right)\right\}_{n=0}^{\infty}$ of connected neighborhoods of infinity in $T_{j}(X)$. For each $n$,

- choose a path $\alpha_{n}$ in $U_{n}$ from $p_{n}$ to $j\left(p_{n}\right)$,

- let $i_{n+1}^{\prime}:\left(U_{n+1}, p_{n}\right) \hookrightarrow\left(U_{n}, p_{n}\right)$ be the inclusion map,

- let $H_{n}=i_{n+1 \#}^{\prime}\left(\pi_{1}\left(U_{n+1}, p_{n}\right)\right)$ and $H_{n}^{\prime}=\hat{\alpha}_{n}\left(\left.j\right|_{\#}\left(\pi_{1}\left(U_{n+1}, p_{n}\right)\right)\right.$ be subgroups of $G_{n}$ and $\phi_{n}: H_{n} \rightarrow H_{n}^{\prime}$ the isomorphism induced by $\left.\hat{\alpha}_{n} \circ j\right|_{\#}$ as promised by Corollary 4.2,

- let $\mu_{n}: \pi_{1}\left(T_{j}\left(U_{n}, U_{n+1}\right), p_{n}\right) \rightarrow \pi_{1}\left(T_{j}\left(U_{n-1}, U_{n}\right), p_{n-1}\right)$ be induced by inclusion followed by the change-of-base point isomorphism corresponding to $r_{n-1}$,

- let $\chi_{\alpha_{n}}: \pi_{1}\left(T_{j}\left(U_{n}, U_{n+1}\right), p_{n}\right) \rightarrow G_{n} *_{\phi_{n}}$ be the isomorphism described in Remark 8, and

- let $\mu_{n}^{\prime}$ be the homomorphism induced by the diagram

$$
\begin{gathered}
\pi_{1}\left(T_{j}\left(U_{n-1}, U_{n}\right), p_{n-1}\right) \stackrel{\mu_{n}}{\longleftarrow} \pi_{1}\left(T_{j}\left(U_{n}, U_{n+1}\right), p_{n}\right) \\
\chi_{\alpha_{n-1}} \downarrow \\
G_{n-1} * \chi_{\alpha_{n}} \downarrow \\
\downarrow
\end{gathered}
$$

Then pro- $\pi_{1}\left(T_{j}(X), r\right)$ admits a representative of the form

$$
G_{0} *_{\phi_{0}} \stackrel{\mu_{1}^{\prime}}{\longleftarrow} G_{1} *_{\phi_{1}} \stackrel{\mu_{2}^{\prime}}{\longleftarrow} G_{2} *_{\phi_{2}} \stackrel{\mu_{3}^{\prime}}{\longleftarrow} \ldots
$$

where each $\mu_{i}^{\prime}$ is equal to $\lambda_{i}$ when restricted to $G_{i}$.

To get the level of precision necessary for future arguments, we need an additional refinement to the above representative of pro- $\pi_{1}\left(T_{j}(X), r\right)$. That will require a hypothesis on the homeomorphism $j: X \rightarrow X$; fortunately, that hypothesis is always satisfied in the cases of interest in this paper.

Proposition 4.4. In addition to the setup of Proposition 4.3, assume the existence of a proper homotopy $H$ in $X$ between the base ray $r$ and its image $j \circ r$. By reparameterizing and rechoosing base points, if necessary, assume that $H([n, \infty) \times[0,1]) \subseteq U_{n}$ for all $n$. Assume also that each $\alpha_{n}$ was chosen to be the track $H\left(\left\{p_{n}\right\} \times[0,1]\right)$ of 
the homotopy. Then each bonding homomorphism $\mu_{n}^{\prime}$ in Proposition 4.3 takes the associated subgroups $H_{n}$ and $H_{n}^{\prime}$ into $H_{n-1}$ and $H_{n-1}^{\prime}$, respectively, and takes the stable letter $t_{n}$ to the stable letter $t_{n-1}$.

Proof. It is clear that $\mu_{n}^{\prime}$ takes $H_{n}$ into $H_{n-1}$. To see that $\mu_{n}^{\prime}$ takes $H_{n}^{\prime}$ into $H_{n-1}^{\prime}$, we must take note of the base paths used. An arbitrary element of $H_{n}^{\prime}$ may be represented by a loop of the form $\alpha_{n} *(j \circ \gamma) * \alpha_{n}^{-1}$, where $\gamma$ is a loop in $U_{n+1}$ based at $p_{n}$. Its image in $\pi_{1}\left(U_{n-1}, p_{n-1}\right)$ is represented by the loop $r_{n-1} * \alpha_{n} *(j \circ \gamma) * \alpha_{n}^{-1} * r_{n-1}^{-1}$. Since the restriction $\left.H\right|_{[n-1, n] \times[0,1]}$ provides a homotopy (rel endpoints) in $U_{n}$ between the paths $r_{n-1} * \alpha_{n}$ and $\alpha_{n-1} *\left(j \circ r_{n-1}\right)$, that loop is homotopic in $U_{n-1}\left(\operatorname{rel} p_{n-1}\right)$ to

$$
\alpha_{n-1} *\left(j \circ r_{n-1}\right) *(j \circ \gamma) *\left(j \circ r_{n-1}\right)^{-1} * \alpha_{n-1}^{-1}=\alpha_{n-1} * j \circ\left(r_{n-1} * \gamma * r_{n-1}^{-1}\right) * \alpha_{n-1}^{-1} \text {. }
$$

The latter of the above is clearly an element of $H_{n-1}^{\prime}$.

To see that $\mu_{n}^{\prime}$ takes $t_{n}$ to $t_{n-1}$, recall from Remark 8, that a representative of $t_{n}$ in $T_{j}\left(U_{n}, U_{n+1}\right)$ is obtained by connecting the ends of the mapping torus fiber $\left\{p_{n}\right\} \times[0,1]$ with the path $\alpha_{n}^{-1}$. A homotopy between this loop and the corresponding representative of $t_{n-1}$ is apparent from the (mapped in) annulus that may be assembled from the rectangles: $r_{n-1} \times[0,1]$ (a family of mapping torus fibers) and $\left.H\right|_{[n-1, n] \times[0,1]}$. Since this annulus lies in $T_{j}\left(U_{n-1}, U_{n}\right)$, has boundary components corresponding to $t_{n-1}$ and $t_{n}$, and contains the path $r_{n-1}$ connecting $p_{n-1}$ to $p_{n}$, the result follows.

Remark 9. (1) Proposition 4.4 tells us that each of the bonding homomorphism $\mu_{n}^{\prime}: G_{n} *_{\phi_{n}} \rightarrow G_{n-1} *_{\phi_{n-1}}$ is, in fact, the homomorphism $\bar{\lambda}_{n}$ induced by $\lambda_{n}$ : $G_{n} \rightarrow G_{n-1}$ as described in Lemma 2.1. Hence, under the hypotheses of Proposition 4.3, a representative

$$
G_{0} *_{\phi_{0}} \stackrel{\bar{\lambda}_{1}}{\longleftarrow} G_{1} *_{\phi_{1}} \stackrel{\bar{\lambda}_{2}}{\longleftarrow} G_{2} *_{\phi_{2}} \stackrel{\bar{\lambda}_{3}}{\longleftarrow} \ldots
$$

of pro- $\pi_{1}\left(T_{j}(X), r\right)$ may be obtained which is, algebraically, an induced HNN sequence (as described in $\oint_{2}$ ) obtained from a base sequence

$$
G_{0} \stackrel{\lambda_{1}}{\longleftarrow} G_{1} \stackrel{\lambda_{2}}{\longleftarrow} G_{2} \stackrel{\lambda_{3}}{\longleftarrow} \ldots
$$

representing pro- $\pi_{1}(X, r)$.

(2) An additional property of this particular HNN sequence is that each associated subgroup $H_{i} \leq G_{i} *_{\phi_{i}}$ is precisely the image under $\bar{\lambda}_{i+1}$ (or, equivalently, $\lambda_{i+1}$ ) of $G_{i+1}$.

(3) When the homeomorphism $j$ generates a $\mathbb{Z}$-action as covering transformations on $X$, the extra hypothesis found in Proposition 4.4 is easily seen to be satisfied. Let $p_{0}$ be a base point and $r_{0}:[0,1] \rightarrow X$ be a path from $p_{0}$ to $j\left(p_{0}\right)$. For each integer $n>0$, let $p_{n}=j^{n}\left(p_{0}\right)$ and $r_{n}=j^{n} \circ r_{0}$, a path from $p_{n}$ to $p_{n+1}$. Let $r:[0, \infty) \rightarrow X$ be the ray obtained by gluing these paths together in the obvious manner. By proper discontinuity of the action of $\langle j\rangle$ on $X, r$ is a proper ray. Moreover, a proper homotopy between $r$ an $j \circ r$ is obtained by sliding each point $r(t)$ one unit forward along $r$ to $j(r(t))$; hence, each $\alpha_{n}$ is just $r_{n}$. 
We close this section with a simple special case that will be useful in $₫ 9$.

Lemma 4.5. Let $X$ be a connected and one-ended with stable pro- $\pi_{1}$ and let $G=$ $\check{\pi}_{1}(X, r)$, where $r$ is a proper ray in $X$. Then any homeomorphism $j: X \rightarrow X$ induces an automorphism $\phi: G \rightarrow G$ and $T_{j}(X)$ is a one-ended space with stable fundamental group at infinity and $\check{\pi}_{1}\left(T_{j}(X), r\right) \cong G \rtimes_{\phi} \mathbb{Z}$.

Proof. By Proposition 2.8, the base ray hypothesis of Proposition 4.4 is satisfied, so all of our previous work applies. By stability, we are in position to apply Lemma 2.7. moreover, in the case at hand, the groups $\underline{G}, \underline{H}$ and $\underline{H}^{\prime}$ of that lemma are all canonically isomorphic to $\check{\pi}_{1}(X, r)$. Thus, the HNN extension promised there is a semidirect product isomorphic to $G \rtimes_{\phi} \mathbb{Z}$.

\section{An EXAMPLE}

The following example is of interest because it helps to justify all of the effort that goes into proving Theorem 1.2 .

Example 5. Let $f:\left(\mathbb{S}^{1}, *\right) \rightarrow\left(\mathbb{S}^{1}, *\right)$ be degree 2 map, and let $X^{\prime}$ be the "bi-infinite mapping telescope" of the system

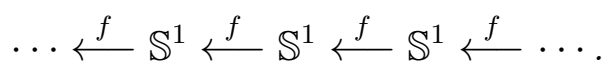

Then let $X$ be the space obtained by adding a single point p to compactify the "left end" of $X^{\prime}$. Since $p$ has arbitrarily small compact contractible neighborhoods in $X$ and since $X$ is locally a 2-dimensional polyhedron at all other points of $X$, it is clear that $X$ is a locally compact ANR. Assemble a proper base ray $r$ from the mapping cylinder arcs corresponding to the base point *. It is easy to see that pro- $\left.\pi_{1}(X, r)\right)$ is represented by the pro-monomorphic system

$$
\mathbb{Z} \stackrel{\times 2}{\longleftarrow} \mathbb{Z} \stackrel{\times 2}{\longleftarrow} \mathbb{Z} \stackrel{\times 2}{\longleftarrow} \cdots .
$$

which is not semistable. Moreover, there is a homeomorphism $j: X \rightarrow X$ that generates a semifree $\mathbb{Z}$-action on $X$. In particular, let $j$ translate each mapping cylinder of $X^{\prime}$ to the right by one and let $j(p)=p$.

In this situation, all of our work in $\$$ 位 valid. With a little effort, one sees that this space $X$ and its mapping torus $T_{j}(X)$ provide a geometric realization of the unusual algebraic situation outlined in Example 4. Since Proposition 1.6 does not apply to actions of this type, there is no contradiction to our theorem.

Remark 10. This example provides a situation where the conclusion of Wright's Theorem 6.3 is satisfied, while the conclusion of our Theorem 1.2 does not hold. Of course, this can happen only because the $\mathbb{Z}$-action is not free.

\section{MANifOld RESUlts}

In this section, manifold means manifold with (possibly empty) boundary. A compact manifold without boundary is called closed and a noncompact manifold without 
boundary is called open. For convenience, all manifolds are assumed to be piecewiselinear. Analogous results may be obtained for smooth or topological manifolds in the usual ways.

Some fundamental facts from manifold topology will be used in this section. First, a Poincaré duality argument implies that a contractible open $n$-manifold $M^{n}$ is always one-ended, provided $n \geq 2$. A deeper result, due to Stallings [St] when $n \geq 5$ and requiring the corresponding Poincaré Conjectures [MT], [Fr] for $n=3,4$, asserts that such a manifold is homeomorphic to $\mathbb{R}^{n}$ if and only if it is simply connected at infinity. By simply connected at infinity, we mean that pro- $\pi_{1}\left(M^{n}, r\right)$ is pro-trivial.

We begin this section with a combined application of Proposition 1.6 and a technique found in Gu2]. The result, which we find interesting in its own right, leads to quick new proofs of two theorems mentioned in the introduction.

Proposition 6.1. Let $J=\langle j\rangle \cong \mathbb{Z}$ act as covering transformations on a contractible open $n$-manifold $M^{n}$ in an orientation preserving manner. Then the mapping torus $T_{j}\left(M^{n}\right)$ is homeomorphic to $\mathbb{R}^{n} \times \mathbb{S}^{1}$.

Proof. For $n \leq 2$ the claim follows from classical results, so assume $n \geq 3$. The quotient space $J \backslash M^{n}$ is an orientable open $n$-manifold homotopy equivalent to $\mathbb{S}^{1}$, so by a Poincaré duality argument (e.g., GT, Prop. 3.1]), $J \backslash M^{n}$ is one-ended. By the techniques of $\$ 3$, a rectangular neighborhood of infinity $R(K \times[u, v])$ in $\left(J \backslash M^{n}\right) \times \mathbb{R}$ will have a fundamental group of the form

$$
\left\langle a, b \mid a^{m}=b^{m}\right\rangle
$$

where $a$ is a generator of $\pi_{1}\left(\left(J \backslash M^{n}\right) \times\{v+1\}\right), b$ is the corresponding generator of $\pi_{1}\left(\left(J \backslash M^{n}\right) \times\{u-1\}\right)$, and $m$ is the index of the image of $\pi_{1}\left(\left(J \backslash M^{n}\right)-K\right)$ in $\pi_{1}\left(J \backslash M^{n}\right)$. Inspection of the proof in Gu2 reveals that, in this particular situation, $\pi_{1}\left(\left(J \backslash M^{n}\right)-K\right)$ surjects onto $\pi_{1}\left(J \backslash M^{n}\right)$. To see that, let $\Sigma^{1} \subseteq J \backslash M^{n}$ be a nicely embedded circle such that inclusion is a homotopy equivalence, and let $N$ be a tubular neighborhood of $\Sigma^{1}$. There is a homotopy of $J \backslash M^{n}$ pulling the entire space into the interior of $N$. Furthermore, there exists a loop lying just outside $N$ that generates $\pi_{1}\left(J \backslash M^{n}\right)$. The techniques found in the proof of [Gu2, Prop. 3.3] show how that loop can be pushed into $\left(J \backslash M^{n}\right)-K$; therefore $\pi_{1}\left(\left(J \backslash M^{n}\right)-K\right) \rightarrow \pi_{1}\left(J \backslash M^{n}\right)$ is surjective. It follows that $\pi_{1}(R(K \times[u, v]))=\left\langle a, b \mid a^{1}=b^{1}\right\rangle=\langle a\rangle=\pi_{1}\left(\left(J \backslash M^{n}\right) \times \mathbb{R}\right)$.

By the above, $\left(J \backslash M^{n}\right) \times \mathbb{R}$ is one-ended with stable infinite cyclic fundamental group at infinity, and inclusion induces an isomorphism

$$
\check{\pi}_{1}\left(\left(J \backslash M^{n}\right) \times \mathbb{R}, r\right) \stackrel{\cong}{\longrightarrow} \pi_{1}\left(\left(J \backslash M^{n}\right) \times \mathbb{R}\right) .
$$

If we let $N^{\prime}$ be a tubular neighborhood of $\Sigma^{1} \times\{0\}$ in $\left(J \backslash M^{n}\right) \times \mathbb{R}$ and $W^{n+1}=$ $\left(\left(J \backslash M^{n}\right) \times \mathbb{R}\right)-i n t\left(N^{\prime}\right)$, then a standard algebraic topology argument (use excision, the Hurewicz Theorem, and the Whitehead Theorem in the universal cover) reveals that $\partial W^{n+1} \hookrightarrow W^{n+1}$ is a homotopy equivalence. By Siebenmann's Open Collar Theorem, when $n+1 \geq 5$, or Gu1, when $n+1=4$, it follows that $W^{n+1} \approx$ $\partial W^{n+1} \times[0, \infty)$. Therefore, $\left(J \backslash M^{n}\right) \times \mathbb{R}$ is homeomorphic to $N^{\prime}$ with an open collar 
attached to its boundary. Since $\left(J \backslash M^{n}\right) \times \mathbb{R}$ is orientable, that space is homeomorphic to $\mathbb{R}^{n} \times \mathbb{S}^{1}$. The result now follows from Proposition 1.6.

The above result is striking when applied, for example, to the exotic contractible open manifolds $M^{n}$ constructed in [Da]. Those manifolds have very large fundamental group at infinity, yet they admit cocompact actions as covering transformations by groups $G$ that are finite index subgroups of infinite Coxeter groups. Certainly, the mapping torus of $\mathrm{id}_{M^{n}}$ is homeomorphic to $M^{n} \times \mathbb{S}^{1}$; and thus maintains a complicated fundamental group at infinity of the form pro- $\pi_{1}\left(M^{n}, r\right) \times \mathbb{Z}$. However, every nontrivial $g \in G$ generates a $\mathbb{Z}$-action on $M^{n}$ so by the above Proposition, whenever $g$ is orientation-preserving, $T_{g}\left(M^{n}\right)$ is topologically just $\mathbb{R}^{n} \times \mathbb{S}^{1}$. This observation can be used to obtain a new proof of the following theorem from [GM] that was reproved in My2.

Theorem 6.2. Let $M^{n}$ be a contractible open n-manifold not homeomorphic to $\mathbb{R}^{n}$ and suppose a group $G$ acts as covering transformations on $M^{n}$. Then the homomorphism $G \rightarrow \mathcal{H}\left(M^{n}\right)$ is injective, where $\mathcal{H}\left(M^{n}\right)$ denotes the group of isotopy classes of self-homeomorphisms of $M^{n}$. In fact, $G \rightarrow \mathcal{W H}\left(M^{n}\right)$ is injective, where $\mathcal{W H}\left(M^{n}\right)$ denotes the group of proper homotopy classes of self-homeomorphisms of $M^{n}$.

Proof. By the hypothesis, $n \geq 3$. Suppose some non-trivial $g \in G$ lies in the kernel of one of the above homomorphisms. By an algebraic topology argument, such a $g$ must be orientation preserving. (Alternatively, that issue can be avoided by considering $g^{2}$.) Since $g$ is non-trivial, Proposition 6.1 asserts that, $T_{g}\left(M^{n}\right)$ is homeomorphic to $\mathbb{R}^{n} \times \mathbb{S}^{1}$. On the other hand, since $g$ is properly homotopic to $\mathrm{id}_{M^{n}}$, that mapping torus is proper homotopy equivalent to $M^{n} \times \mathbb{S}^{1}$. Since the pro-fundamental group is an invariant of proper homotopy type, this can happen only if $M^{n}$ is simply-connected at infinity, contradicting the hypothesis that it is not homeomorphic to $\mathbb{R}^{n}$.

We now employ our methods to obtain a new proof of:

Theorem 6.3 (Wright's Main Theorem). Let $M^{n}$ be a contractible open $n$-manifold with pro-monomorphic fundamental group at infinity. If $M^{n}$ admits a nontrivial action by covering transformations, then $M^{n} \approx \mathbb{R}^{n}$.

Proof. Again we may assume $n$ is at least 3. Any group acting by covering transformations on a contractible manifold is torsion free; thus, $M^{n}$ admits such an action by an infinite cyclic group $J=\langle j\rangle$. By passing to an index two subgroup if necessary, assume that the elements of $J$ are orientation preserving homeomorphisms.

By our work in 84 , pro- $\pi_{1}\left(T_{j}\left(M^{n}\right), r\right)$ has a representative of the form

$$
G_{0} *_{\phi_{0}} \stackrel{\bar{\lambda}_{1}}{\longleftarrow} G_{1} *_{\phi_{1}} \stackrel{\bar{\lambda}_{2}}{\longleftarrow} G_{2} *_{\phi_{2}} \stackrel{\bar{\lambda}_{3}}{\longleftarrow} \ldots
$$

where

$$
G_{0} \stackrel{\lambda_{1}}{\longleftarrow} G_{1} \stackrel{\lambda_{2}}{\longleftarrow} G_{2} \stackrel{\lambda_{3}}{\longleftarrow} \ldots
$$

is a representative of pro- $\pi_{1}\left(M^{n}, r\right)$. On the other hand, Proposition 6.1 tells us that pro- $\pi_{1}\left(T_{j}\left(M^{n}\right), r\right)$ is pro-isomorphic to the constant sequence $\{\mathbb{Z}, \mathrm{id}\}$. After passing 
to subsequences there exists a ladder diagram of the form

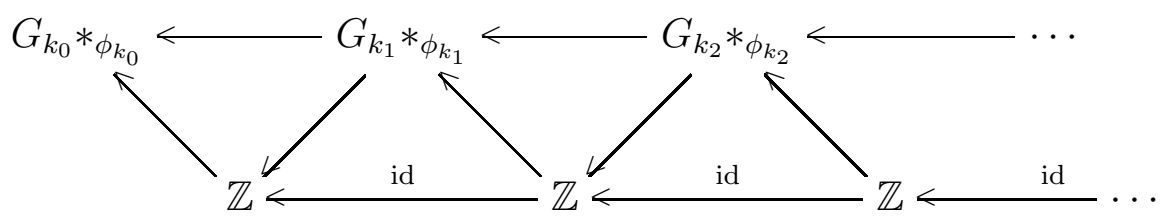

From this diagram, we may conclude that each homomorphism in the top row has an infinite cyclic image. Since these homomorphisms send stable letter to stable letter. It follows that the image of each $G_{k_{i+1}}$ in $G_{k_{i}}$ is trivial. Thus, the representative

$$
G_{k_{0}} \longleftarrow G_{k_{1}} \longleftarrow G_{k_{2}} \longleftarrow \cdots
$$

of pro- $\pi_{1}\left(M^{n}, r\right)$ is pro-trivial; so $M^{n}$ is simply connected at infinity and, thus, homeomorphic to $\mathbb{R}^{n}$.

\section{Proof of Theorem 1.2; The Algebraic PART}

We now begin the proof of Theorem 1.2. With Theorem 1.1 as a starting point, we need only prove:

Proposition 7.1. Let $X$ be a one-ended simply-connected, locally compact and locally path connected separable metric space with pro-monomorphic fundamental group at infinity. If $\mathbb{Z}$ acts by covering transformations on $X$, then $X$ has stable fundamental group at infinity.

The proof is more intricate, but similar in spirit to our proof of Theorem 6.3. By work done in $\$ 4$, there exists a proper ray $r$ in $X$, a representative

$$
G_{0} \stackrel{\lambda_{1}}{\longleftarrow} G_{1} \stackrel{\lambda_{2}}{\longleftarrow} G_{2} \stackrel{\lambda_{3}}{\longleftarrow} \cdots
$$

of pro- $\pi_{1}(X, r)$, subgroups $H_{i}, H_{i}^{\prime} \leq G_{i}$, and isomorphisms $\phi_{i}: H_{i} \rightarrow H_{i}^{\prime}$ with $\phi_{i-1} \circ$ $\lambda_{i}(h)=\lambda_{i} \circ \phi_{i}(h)$ for all $h \in H_{i}$, so that the corresponding induced HNN sequence

$$
G_{0} *_{\phi_{0}} \stackrel{\bar{\lambda}_{1}}{\longleftarrow} G_{1} * \phi_{1} \stackrel{\bar{\lambda}_{2}}{\longleftarrow} G_{2} *_{\phi_{2}} \stackrel{\bar{\lambda}_{3}}{\longleftarrow} \ldots
$$

represents pro- $\pi_{1}\left(T_{j}(X), r\right)$, where $j: X \rightarrow X$ is a generator of the $\mathbb{Z}$-action on $X$.

Our work in $\$ 3$ provides, for an appropriately chosen base ray $r^{\prime}$, a representative of pro- $\pi_{1}\left((J \backslash X) \times \mathbb{R}, r^{\prime}\right)$ of the form

$$
\Theta_{0} \stackrel{\rho_{1}}{\longleftarrow} \Theta_{1} \stackrel{\rho_{2}}{\longleftarrow} \Theta_{2} \stackrel{\rho_{3}}{\longleftarrow} \ldots
$$

where the $\Theta_{i}$ and the $\rho_{i}$ are as described in that section.

To prove Proposition 7.1, we need to show that sequence (7.1) is semistable. By Proposition 1.6 we know that $(J \backslash X) \times \mathbb{R} \approx T_{j}(X)$. If a homeomorphism $h:(J \backslash X) \times$ $\mathbb{R} \rightarrow T_{j}(X)$ can be chosen which sends an appropriately chosen base ray $r^{\prime}$ in $(J \backslash X) \times$ $\mathbb{R}$ (as described in Proposition 3.1) to an appropriate base ray $r$ in $T_{j}(X)$ (as described in Proposition 4.4 and the remark that follows it) then the sequences (7.2) and (7.3) are pro-isomorphic. Assuming this for the moment, a quick conclusion to our proof might be expected as follows: First use the (explicit) semistability of (7.3) to conclude that (7.2) is semistable, then use the semistability of (7.2) to conclude that (17.1) is 
semistable. Unfortunately, Example 4 shows that the second of these implications is not automatic. Instead, we require a more delicate argument that relies on "normal forms" for HNN extensions and some special properties of the groups and diagrams at hand.

We save for the following section, an investigation of the natural homeomorphism between $(J \backslash X) \times \mathbb{R}$ and $T_{j}(X)$, where it will be observed that there is no problem with base rays - and hence, there exist subsequences of (7.2) and (7.3) and a commutative diagram of the form:

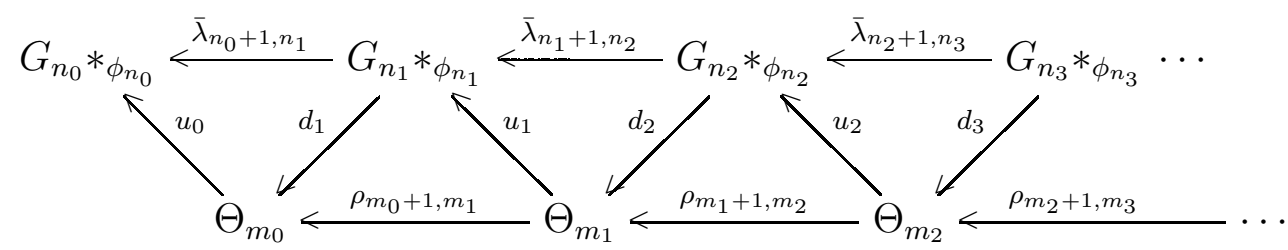

That investigation will also show that each $u_{i}$ can be arranged to take the preferred generator $a \in \Theta_{m_{i}}$ to the stable letter $t_{n_{i}} \in G_{n_{i}} *_{\phi_{n_{i}}}$. For now, we assume those arrangements have been made and proceed with the algebraic part of the proof.

By our work in $\oint 3$, for every $i$, there is an integer $N_{m_{i}}>0$ for which $a^{N_{m_{i}}}$ lies in the center of $\Theta_{m_{i}}$; therefore, $t_{n_{i}}^{N_{m_{i}}}$ is central in $\operatorname{Im} u_{i}=\operatorname{Im} \bar{\lambda}_{n_{i}+1, n_{i+1}}$. For each $i$, let $K_{i} \leq G_{n_{i}}$ be the image of $G_{n_{i+1}}$ under $\bar{\lambda}_{n_{i}+1, n_{i+1}}$ and note that $\operatorname{Im} \bar{\lambda}_{n_{i}+1, n_{i+1}}$ is precisely the subgroup of $G_{n_{i}} *_{\phi_{n_{i}}}$ generated by $K_{i}$ together with the stable letter $t_{n_{i}}$. We indicate this by writing $\operatorname{Im} \bar{\lambda}_{n_{i}+1, n_{i+1}}=\left\langle K_{i}, t_{n_{i}}\right\rangle$. Then, by semistability, we may assume (after passing to a further subsequence and relabeling) that each of the bonds in the corresponding pro-equivalent inverse sequence is surjective.

$$
\left\langle K_{0}, t_{n_{0}}\right\rangle \leftarrow\left\langle K_{1}, t_{n_{1}}\right\rangle \nleftarrow\left\langle K_{2}, t_{n_{2}}\right\rangle \leftarrow\left\langle K_{3}, t_{n_{3}}\right\rangle \nleftarrow \cdots
$$

For the moment let $i$ be fixed and, to simplify notation, let $N$ denote $N_{m_{i}}$ and $t$ denote $t_{n_{i}}$. Then $t^{N}$ is central in $\left\langle K_{i}, t\right\rangle$, so $t^{-N} K_{i} t^{N}=K_{i}$; and since $K_{i} \leq H_{n_{i}}$, an application of the standard relators in $G_{n_{i}} *_{\phi_{n_{i}}}$ shows that $t^{-(N-1)} \phi_{n_{i}}\left(K_{i}\right) t^{N-1}=K_{i}$. If $N=1$ this means $\phi_{n_{i}}$ is an automorphism of $K_{i}$; otherwise a normal forms argument implies that $\phi_{n_{i}}\left(K_{i}\right) \leq H_{n_{i}}$ and $t^{-(N-2)} \phi_{n_{i}}^{2}\left(K_{i}\right) t^{N-2}=K_{i}$. Continuing inductively, we deduce that $\phi_{n_{i}}^{q-1}\left(K_{i}\right) \leq H_{m_{i}}$ and $t^{-(N-q)} \phi_{n_{i}}^{q}\left(K_{i}\right) t^{N-q}=K_{i}$ for all $1 \leq q \leq N$, with the final observation being that $\phi_{n_{i}}^{N}\left(K_{i}\right)=K_{i}$. From this it is easy to see that conjugating by any (positive or negative) power of $t$ simply permutes the subgroups of $H_{m_{i}}$ in the collection $\left\{\phi_{n_{i}}^{r}\left(K_{i}\right)\right\}_{r=0}^{N-1}$. And if we let $K_{i}^{\prime}=\left\langle K_{i}, \phi_{n_{i}}\left(K_{i}\right), \phi_{n_{i}}^{2}\left(K_{i}\right), \cdots, \phi_{n_{i}}^{N-1}\left(K_{i}\right)\right\rangle$, the subgroup of $H_{n_{i}}$ generated by these groups, then $\left\langle K_{i}, t\right\rangle=K_{i}^{\prime} \cdot\langle t\rangle$. Since the latter two groups intersect trivially and $K_{i}^{\prime}$ is normal in $\left\langle K_{i}, t\right\rangle$, it follows that $\left\langle K_{i}, t\right\rangle$ is a semidirect product $K_{i}^{\prime} \rtimes\langle t\rangle$.

Rewrite (7.5) as

$$
K_{0}^{\prime} \rtimes\left\langle t_{n_{0}}\right\rangle \leftarrow K_{1}^{\prime} \rtimes\left\langle t_{n_{1}}\right\rangle \nleftarrow K_{2}^{\prime} \rtimes\left\langle t_{n_{2}}\right\rangle \leftarrow K_{3}^{\prime} \rtimes\left\langle t_{n_{3}}\right\rangle \nleftarrow \cdots
$$

and recall that each bonding homomorphism takes $t_{n_{i+1}}$ to $t_{n_{i}}$ and $K_{i+1}^{\prime}$ into $K_{i}^{\prime}$. Such homomorphisms can be surjective only if the $K_{i+1}^{\prime}$ surject onto $K_{i}^{\prime}$. Moreover, since 
$K_{i+1}^{\prime} \leq H_{n_{i}+1} \leq G_{n_{i+1}}$ and $K_{i}$ is the image of $G_{n_{i+1}}$ under $\bar{\lambda}_{n_{i}+1, n_{i+1}}$, it follows that $K_{i}^{\prime}=K_{i}$. This provides an inverse sequence of surjections

$$
K_{0} \nleftarrow K_{1} \leftarrow K_{2} \nleftarrow K_{3} \nleftarrow \cdots
$$

which is pro-equivalent to (7.1), as desired.

\section{Proof of Theorem 1.2; Topological Details}

We now lay out the topological argument needed to complete the proof of Proposition 7.1 and, thus, Theorem 1.2. Our remaining task is to prove the existence of a ladder diagram of the form of 7.4 with the additional property that $u_{i}(a)=t_{n_{i}}$ for each $i$.

The process described at the end of Section 2 applied to a homeomorphism $h$ : $(J \backslash X) \times \mathbb{R} \rightarrow T_{j}(X)$ (as promised in Proposition 1.6), produces a ladder diagram between a subsequences of any given representations of pro- $\pi_{1}((J \backslash X) \times \mathbb{R}, r)$ and pro$\pi_{1}\left(T_{j}(X), h \circ r\right)$. Since the algebraic proof presented above used specific properties of both (7.3) and (7.2), we require a base ray $r$ in $(J \backslash X) \times \mathbb{R}$ of the type specified in Proposition 3.1 whose image $h \circ r$ in $T_{j}(X)$ is of the type described in Remark 9 . That will be accomplished by taking a close look at Proposition 1.6. In doing so, it will also become clear that homomorphisms $u_{i}$ in the resulting diagram take primary generators $a$ to stable letters $t_{n_{i}}$.

Our goal is to "see" the homeomorphism $h:(J \backslash X) \times \mathbb{R} \rightarrow T_{j}(X)$ promised by 7.1. Toward that end, choose a map $F: J \backslash X \rightarrow \mathbb{S}^{1}$ that induces an isomorphism on fundamental groups and let $\pi: X \rightarrow J \backslash X$ be the quotient map. Then $\pi$ is a universal covering map with deck transformations generated by $j$. Let $p: \mathbb{R} \rightarrow \mathbb{S}^{1}$ be the universal covering map with covering transformations generated by unit translation, and choose $f: X \rightarrow \mathbb{R}$ to be a lift of $F \circ \pi$. For any $A \subseteq \mathbb{R}$, let $X_{A}=f^{-1}(A)$. Note that, for any unit interval $[y, y+1] \subseteq \mathbb{R}$, the restriction of $\pi$ to $X_{[y, y+1]}$ is a quotient map that creates a copy of $J \backslash X$ by identifying each $x \in X_{y}$ with $j(x) \in X_{y+1}$.

Consider the diagram,

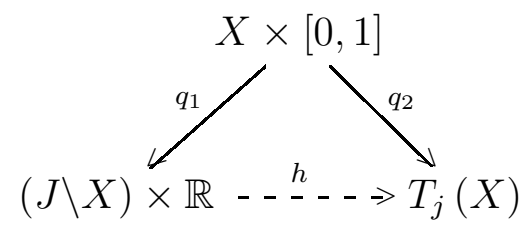

where $q_{1}(x, u)=(\pi(x), f(x)+u)$ and $q_{2}$ is the quotient map that defines $T_{j}(X)$; specifically, $(x, 1)$ is identified with $(j(x), 0)$ for each $x \in X$. Note that $q_{1}(x, u)=$ $q_{1}\left(x^{\prime}, u^{\prime}\right)$ if and only if $\pi(x)=\pi\left(x^{\prime}\right)$ and $f(x)+u=f\left(x^{\prime}\right)+u^{\prime}$. The first of those conditions implies that $\left|f(x)-f\left(x^{\prime}\right)\right|$ is an integer; hence, $\left|u-u^{\prime}\right|$ is an integer. It follows that $u=u^{\prime}$ or (without loss of generality) $u=0$ and $u^{\prime}=1$. In the first case $f(x)=f\left(x^{\prime}\right)$, implying that $(x, u)=\left(x^{\prime}, u^{\prime}\right)$. In the latter case, $f(x)=f\left(x^{\prime}\right)+1$ and, since $\pi(x)=\pi\left(x^{\prime}\right)$, this implies that $j\left(x^{\prime}\right)=x$. Thus $(x, 0)$ may be viewed as $\left(j\left(x^{\prime}\right), 0\right)$. We conclude that, when a pair of points in $X \times[0,1]$ is identified under $q_{1}$, it also identified under $q_{2}$. A similar argument gives the converse; therefore diagram (8.1) induces a homeomorphism $h:(J \backslash X) \times \mathbb{R} \rightarrow T_{j}(X)$. 


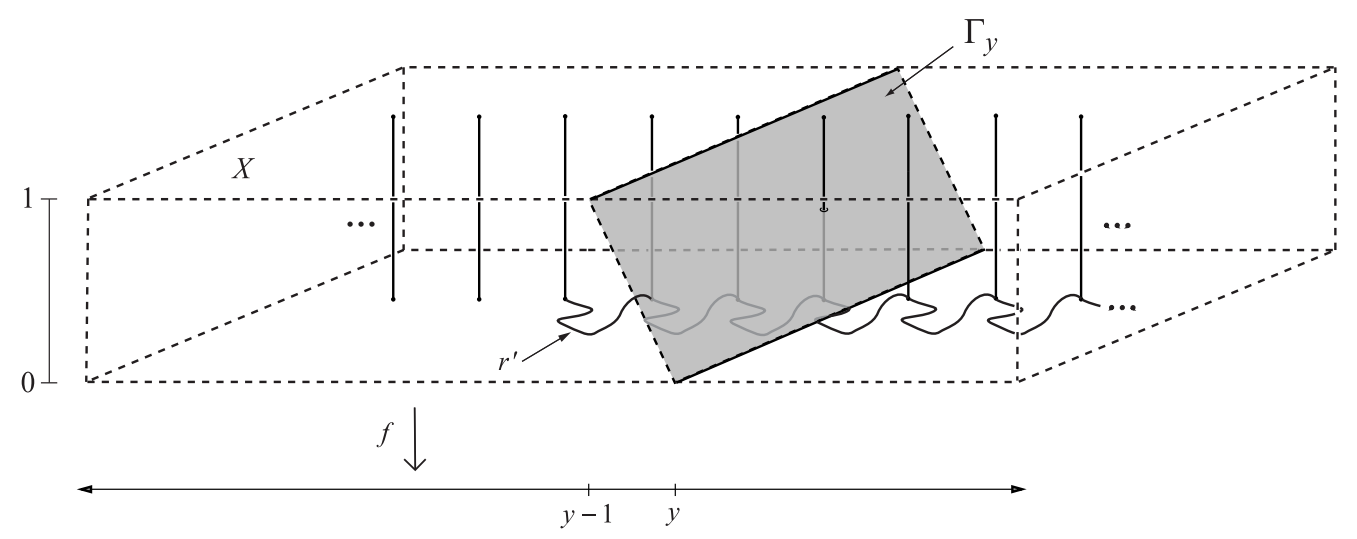

Figure 2. Preimages of $(J \backslash X) \times\{r\}$ and $\{b\} \times \mathbb{R}$ under $q_{1}$.

Remark 11. The above does not require simple connectivity of $X$; the construction can be carried out more generally by choosing $F: J \backslash X \rightarrow S^{1}$ to induce the epimorphism $\pi_{1}(J \backslash X, b) \rightarrow \mathbb{Z}$ with kernel equal to $\pi_{\#}\left(\pi_{1}(X, e)\right)$, where $e \in \pi^{-1}(b)$. This provides the elementary proof of Proposition 1.6 promised in the introduction.

Diagram (8.1) provides a common space, $X \times[0,1]$, with which to compare the product structure of $(J \backslash X) \times \mathbb{R}$ with the mapping torus structure of $T_{j}(X)$. The latter is easy to visualize; one simply glues the top edge $X \times\{1\}$ to the bottom $X \times\{0\}$ via a shift that identifies $(x, 1)$ with $(j(x), 0)$. To see the product structure of $(J \backslash X) \times \mathbb{R}$, we look at the preimages under $q_{1}$ of factor spaces $(J \backslash X) \times\{y\}$ and $\{b\} \times \mathbb{R}$. For fixed $y \in \mathbb{R}$,

$$
q_{1}^{-1}((J \backslash X) \times\{y\})=\{(x, u) \mid u=y-f(x)\},
$$

which may be viewed as the portion of the graph of the function $(-f)+y: X \rightarrow \mathbb{R}$ lying between $u=0$ and $u=1$. Call this set $\Gamma_{y}$ and note that it lies entirely within $X_{[y-1, y]} \times[0,1]$. Viewed differently, it is homeomorphic to the portion of the graph of $f$ lying between $u=y-1$ and $u=y$ and thus is homeomorphic to $X_{[y-1, y]}$. Moreover, under that homeomorphism, the identifications made on $\Gamma_{y}$ via $q_{1}$ correspond to the identifications made to $X_{[y-1, y]}$ under $\pi: X \rightarrow J \backslash X$; both yield copies of $J \backslash X$. For fixed $b \in J \backslash X$

$$
\begin{aligned}
q_{1}^{-1}((\{b\} \times \mathbb{R}) & =\{(x, u) \mid \pi(x)=b\} \\
& =\pi^{-1}(b) \times[0,1] \\
& =\left\{j^{k}(e)\right\}_{k \in \mathbb{Z}} \times[0,1],
\end{aligned}
$$

where $e \in \pi^{-1}(b)$. Under the quotient map $q_{1}$, the line $\{b\} \times \mathbb{R}$ is assembled from $\left\{j^{k}(e)\right\}_{k \in \mathbb{Z}} \times[0,1]$ by identifying the top endpoint of each $j^{k}(e) \times[0,1]$ with the bottom endpoint of $j^{k+1}(p) \times[0,1]$. See Figure 2 .

In order to obtain the desired rays $r$ and $h \circ r$ in $(J \backslash X) \times \mathbb{R}$ and $T_{j}(X)$, we construct a single proper ray $r^{\prime}$ in $X=X \times\{0\} \subseteq X \times[0,1]$ and let $r=q_{1} \circ r^{\prime}$; then $h \circ r$ is 
precisely $q_{2} \circ r^{\prime}$. Following the prescription found in Remark 9, let $p_{0} \in X_{\{1\}}$ be a base point and $r_{0}^{\prime}:[0,1] \rightarrow X$ a path from $p_{0}$ to $j\left(p_{0}\right) \in X$; for each $n>0$, let $p_{n}=j^{n}\left(p_{0}\right)$ and $r_{n}^{\prime}=j^{n} \circ r_{0}^{\prime}$ (a path from $p_{n}$ to $p_{n+1}$ ). Obtain $r^{\prime}:[0, \infty) \rightarrow X$ by gluing these paths together in the obvious manner. See Figure 2, By choosing the initial path $r_{0}^{\prime}$ to lie in $X_{(0, \infty)}$, it will follow that $r^{\prime}([n, \infty)) \subseteq X_{(n, \infty)}$ for all integers $n \geq 0$. Since $q_{1}\left(X_{(n, \infty)} \times\{0\}\right) \subseteq(J \backslash X) \times(n, \infty)$, it follows that $r([n, \infty)) \subseteq(J \backslash X) \times(n, \infty)$ for all $n$; so $r$ is in accordance with Proposition 3.1. By construction, $q_{2} \circ r^{\prime}$ fits the specifications of Remark 9, so the base ray issue is resolved - there is a ladder diagram of type (7.4).

Lastly we observe that each $u_{i}$ in (7.4) takes the primary generator $a \in \Theta_{m_{i}}$ to the stable letter $t_{n_{i}} \in G_{n_{i}} *_{\phi_{n_{i}}}$. Following the discussion found in Remarks 8 and 9 , $t_{n_{i}}$ may be represented by the loop in $T_{j}\left(U_{n_{i}}, U_{n_{i}+1}\right)$ which is a concatenation of the $q_{2}$-image of the interval $p_{n_{i}+1} \times[0,1]$ with the path $\left(\left.r^{\prime}\right|_{\left[n_{i+1}, n_{i+1}+1\right]}\right)^{-1}$ in $X \times\{0\}$. The $q_{1}$-images of these paths lie in $(J \backslash X) \times\left[n_{i}, \infty\right)$ where the resulting loop is easily seen to generate $\pi_{1}\left((J \backslash X) \times\left[n_{i}, \infty\right)\right)$. This is the primary generator $a$ of a rectangular neighborhood of infinity of the form $R\left(K_{n_{i}} \times\left[-n_{i}, n_{i}\right]\right)$. Thus, $h$ takes this representation of $a$ to $t_{n_{i}}$. Since, each $u_{i}$ is induced by a restriction of the homeomorphism $h$, and since we have arranged that all bonding homomorphisms in (7.3) and (7.2) take primary generator to primary generator and and stable letter to stable letter, it follows that each $u_{i}$ takes $a$ to $t_{n_{i}}$.

\section{ACtions By $\mathbb{Z} \oplus \mathbb{Z}$}

In this section we prove Theorem 1.3. Cocompact actions of $\mathbb{Z} \oplus \mathbb{Z}$ are wellunderstood, so we discuss only the non-cocompact case. We will prove the following:

Theorem 9.1. Let $X$ be a one-ended, simply connected, locally compact ANR with pro-monomorphic fundamental group at infinity. If $X$ admits a $\mathbb{Z} \oplus \mathbb{Z}$ action by covering transformations that is not cocompact, then $X$ is simply connected at infinity.

Proof. Assume $G \cong \mathbb{Z} \oplus \mathbb{Z}$ acts by covering transformations on $X$. Write $G=\left\langle j_{1}\right\rangle \oplus$ $\left\langle j_{2}\right\rangle$ where each $j_{i}$ is a self-homeomorphism of $X$. By Proposition 1.6. $\left(\left\langle j_{1}\right\rangle \backslash X\right) \times \mathbb{R} \approx$ $T_{j_{1}}(X)$; moreover, we will see that $j_{2}$ induces natural self-homeomorphisms of each of these spaces. This is largely due to the fact that $j_{2} j_{1}=j_{1} j_{2}$.

Let $\sim$ be the equivalence relation on $X$ induced by the action of $\left\langle j_{1}\right\rangle$ and let $[x]$ denote a corresponding equivalence class. Then $\breve{j}_{2}:\left\langle j_{1}\right\rangle \backslash X \rightarrow\left\langle j_{1}\right\rangle \backslash X$ defined by $\breve{j}_{2}([x])=\left[j_{2}(x)\right]$ is a well-defined function. Indeed, if $x \sim y$ then $y=j_{1}^{k}(x)$ for some integer $k$. Then $j_{2}(y)=j_{2} j_{1}^{k}(x)=j_{1}^{k} j_{2}(x)$, and the last of these terms is equivalent to $j_{2}(x)$ by definition. Continuity of $\breve{j}_{2}$ is clear; moreover, $j_{2}^{-1}$ induces a continuous inverse for $\breve{j}_{2}$ in an analogous manner. Thus, $\breve{j}_{2}$ is a homeomorphism. Let $\bar{j}_{2}=\left(\breve{j}_{2}, \operatorname{id}_{\mathbb{R}}\right):\left(\left\langle j_{1}\right\rangle \backslash X\right) \times \mathbb{R} \rightarrow\left(\left\langle j_{1}\right\rangle \backslash X\right) \times \mathbb{R}$.

The desired self-homeomorphism of $T_{j_{1}}(X)=X \times[0,1] /\left\{(x, 1) \sim\left(j_{1}(x), 0\right)\right\}$ is obtained by letting $j_{2}$ act on each slice $X \times\{t\}$. Since $j_{2}\left(j_{1}(x)\right)=j_{1}\left(j_{2}(x)\right)$, applying $j_{2}$ to a pair of equivalent points $(x, 1)$ and $\left(j_{1}(x), 0\right)$ yields a pair of equivalent 
points $\left(j_{2}(x), 1\right)$ and $\left(j_{1} j_{2}(x), 0\right)$. Let $\underline{j}_{2}: T_{j_{1}}(X) \rightarrow T_{j_{1}}(X)$ be the resulting homeomorphism.

A quick check of diagram 8.1 shows that the induced homeomorphism $h$ is equivariant with respect to the $\mathbb{Z}$-actions induced by $\bar{j}_{2}$ and $\underline{j}_{2}$. So, by a second application of Proposition 1.6, we get

$$
\left\langle\bar{j}_{2}\right\rangle \backslash\left(\left(\left\langle j_{1}\right\rangle \backslash X\right) \times \mathbb{R}\right) \times \mathbb{R} \approx T_{\underline{j}_{2}}\left(T_{j_{1}}(X)\right) .
$$

We are now prepared to employ our standard strategy; in particular, we will use identity (9.1) to obtain a pair of inverse sequences representing pro-fundamental groups of these spaces. Comparison of those sequences will reveal the desired conclusion.

The left-hand side of (9.1) is easily seen to be homeomorphic to

$$
\left(\left(\left\langle j_{1}\right\rangle \oplus\left\langle j_{2}\right\rangle\right) \backslash X\right) \times \mathbb{R}^{2}
$$

The space $\left(\left\langle j_{1}\right\rangle \oplus\left\langle j_{2}\right\rangle\right) \backslash X$ has fundamental group isomorphic to $\left\langle j_{1}\right\rangle \oplus\left\langle j_{2}\right\rangle$. It is a standard fact that, given a path connected noncompact space $Y$, the "doubly stabilized" product $Y \times \mathbb{R}^{2}$, has a stable fundamental group at infinity isomorphic to $\pi_{1}(Y)$. To see this, first note that the noncompactness of $Y$ implies that $Y \times \mathbb{R}$ is oneended; moreover, the fundamental group of each neighborhood of that end surjects onto $\pi_{1}(Y \times \mathbb{R})$. So the techniques of $\$ 3$, applied to $(Y \times \mathbb{R}) \times \mathbb{R}$, show that this space has arbitrarily small rectangular neighborhoods of infinity with fundamental group described by a graph of groups with just two vertices and one edge - all labeled by $\pi_{1}(Y)$.

To understand the fundamental group at infinity for $T_{\underline{j}_{2}}\left(T_{j_{1}}(X)\right)$, first use Theorem 1.2 to deduce that $X$ has a stable finitely generated free fundamental group at infinity. If $\check{\pi}_{1}(X, r)=F$, where $F$ is a finitely generated free group, then by Lemma 4.5, $T_{j_{1}}(X)$ has stable pro- $\pi_{1}$ isomorphic to a semidirect product $F \rtimes \mathbb{Z}$. Applying Lemma 4.5 a second time, we see that $T_{\underline{j}_{2}}\left(T_{j_{1}}(X)\right)$ has a stable fundamental group at infinity of the form $(F \rtimes \mathbb{Z}) \rtimes \mathbb{Z}$.

Combining the above observations, we have $(F \rtimes \mathbb{Z}) \rtimes \mathbb{Z} \cong\left\langle j_{1}\right\rangle \oplus\left\langle j_{2}\right\rangle \cong \mathbb{Z} \oplus \mathbb{Z}$. When a semidirect product is abelian, the factor groups must both be abelian and the product an ordinary direct product. It follows that $F \rtimes \mathbb{Z}=F \times \mathbb{Z}$ and $(F \rtimes \mathbb{Z}) \rtimes$ $\mathbb{Z}=(F \times \mathbb{Z}) \times \mathbb{Z}$. The latter group is isomorphic to $\mathbb{Z} \oplus \mathbb{Z}$ if and only if $F$ is the trivial group. Hence, $X$ is simply connected at infinity.

\section{REFERENCES}

[Bo] B.H. Bowditch, Planar groups and the Seifert conjecture, J. Reine Angew. Math. 576 (2004), $11-62$.

[Co] D.E. Cohen, Combinatorial Group Theory: a topological approach, London Mathematical Society Student Texts, 14. Cambridge University Press, Cambridge, 1989. x+310 pp.

[Da] M. Davis, Groups generated by reflections and aspherical manifolds not covered by Euclidean spaces, Ann. Math. 117 (1983), 293-324.

[Fa] F.T. Farrell, The second cohomology group of $G$ with $\mathbb{Z}_{2} G$ coefficients, Topology 13 (1974), 313-326.

[Fr] M.H. Freedman, The topology of four-dimensional manifolds, J. Differential Geom. 17 (1982), no. $3,357-453$. 
[GM] R. Geoghegan and M. Mihalik, The fundamental group at infinity, Topology 35 (1996), 655669.

[Ge] R. Geoghegan, Topological methods in group theory, Graduate Texts in Mathematics, 243. Springer, New York, 2008. xiv+473 pp.

[GO] R. Geoghegan and P. Ontaneda, Boundaries of cocompact proper CAT(0) spaces, Topology 46 (2007), 129-137.

[GT] C.R. Guilbault and F.C. Tinsley, Manifolds with non-stable fundamental groups at infinity. II, Geom. Topol. 7 (2003), 255-286.

[Gu1] C.R. Guilbault, An open collar theorem for 4-manifolds, Trans. Amer. Math. Soc. 331 (1992), no. $1,227-245$.

[Gu2] C.R. Guilbault, Products of open manifolds with $\mathbb{R}$, Fund. Math. 197 (2007), 197-214.

[Mc] D.R. McMillan, Some contractible open 3-manifolds, Trans. Amer. Math. Soc. 1021962 373382.

[Me] J. Meier, Groups, graphs and trees. An introduction to the geometry of infinite groups, London Mathematical Society Student Texts, 73. Cambridge University Press, Cambridge, 2008. xii+231 pp.

[LS] R.C. Lyndon and P.E. Schupp, Combinatorial group theory. Reprint of the 1977 edition. Classics in Mathematics. Springer-Verlag, Berlin, 2001. xiv+339 pp.

[MT] J. Morgan and G. Tian, Ricci flow and the Poincaré conjecture, Clay Mathematics Monographs, 3. American Mathematical Society, Providence, RI; Clay Mathematics Institute, Cambridge, MA, 2007. xlii+521 pp.

[My1] R. Myers, Contractible open 3-manifolds which are not covering spaces, Topology 27 (1988), $27-35$.

[My2] R. Myers, On covering translations and homeotopy groups of contractible open 3-manifolds, Proc. Amer. Math. Soc. 128 (2000), no. 5, 1563-1566.

[Si] L.C. Siebenmann, On detecting open collars, Trans. Amer. Math. Soc. 142 (1969) 201-227.

[St] J. Stallings, The piecewise linear structure of Euclidean space, Proc. Cambridge Philos. Soc., 58 (1962), 481-488.

[Sw] E.L. Swenson, A cut point theorem for CAT(0) groups, J. Differential Geom. 53 (1999), 327358.

[Wh] J.H.C. Whitehead, A certain open manifold whose group is unity, Quarterly J. Math., 6 (1935), 268-279.

[Wr] D.G. Wright, Contractible open manifolds which are not covering spaces, Topology 31 (1992), 281-291.

Department of Mathematical Sciences, Binghamton University, Binghamton, New YORK 13902-6000

E-mail address: ross@math.binghamton.edu

Department of Mathematical Sciences, University of Wisconsin-Milwaukee, MilWAUKEE, WISCONSIN 53201

E-mail address: craigg@uwm.edu 